\title{
Polymorphic and Isomorphic Cocrystals of a N-salicylidene-3-aminopyridine with Dicarboxylic Acids: Tuning of Solid-state Photo- and Thermochromism
}

\author{
Andrea Carletta, ${ }^{\mathrm{a}}$ Xavier Buol, ${ }^{\mathrm{a}}$ Tom Leyssens, ${ }^{\mathrm{b}}$ Benoît Champagne, ${ }^{\mathrm{a}}$ and Johan \\ Wouters*a \\ ${ }^{\mathrm{a} U n i t e ́ ~ d e ~ C h i m i e ~ P h y s i q u e, ~ T h e ́ o r i q u e ~ e t ~ S t r u c t u r a l e, ~ C h e m i s t r y ~ D e p a r t m e n t, ~ U n i v e r s i t y ~ o f ~}$ \\ Namur, 61 rue de Bruxelles, B-5000 Namur, Belgium \\ ${ }^{\mathrm{b}}$ Institute of Condensed Matter and Nanosciences, Université Catholique de Louvain, Louvain- \\ La-Neuve, Belgium \\ E-mail: johan.wouters@unamur.be; Tel: +32 (0)81/72.45.50
}

\section{Supporting Information:}

Figure S1) PXRD of 1

Figure S2) PXRD of 2 and $\mathbf{3}$

Figure S3) PXRD of 4 and 5

Figure S4) Isostructurality between 2 and 4: PXRD and structure matching

Figure S5) ORTEP diagrams

Figure S6) Crystal packing of 1, 4, 5

Figure S7) Histograms of the bond length in anils exhibiting their enol form

Figure S8) Histograms of the bond length in anils exhibiting their cis-keto form

Figure S9) Thermochromic behavior of crystals

Figure S10) UV-Vis silid-state spectra, before and after irradiation, of $\mathbf{1}$ and $\mathbf{5}$

Figure S11) FTIR spectra

Figures S12) and S13) DSC: melting point determination

Table S1) Elemental Analysis of 1

Table S2) Room Temperature SCXRD measurements: crystallographic parameters 
Table S3) Selected Geometries for H-bonds.

Table S4) Selected geometries for carboxyl group in FA and SA.

Table S5) Bond lengths of the six-membered pseudocycle in anils under the enol form

Table S6) Bond lengths of the six-membered pseudocycle in anils under the cis-keto form

Table S7) Selected structures of SCC and MCC used for construction of model based on Hirshfeld surfaces C...C\% contacts calculation. 
a)

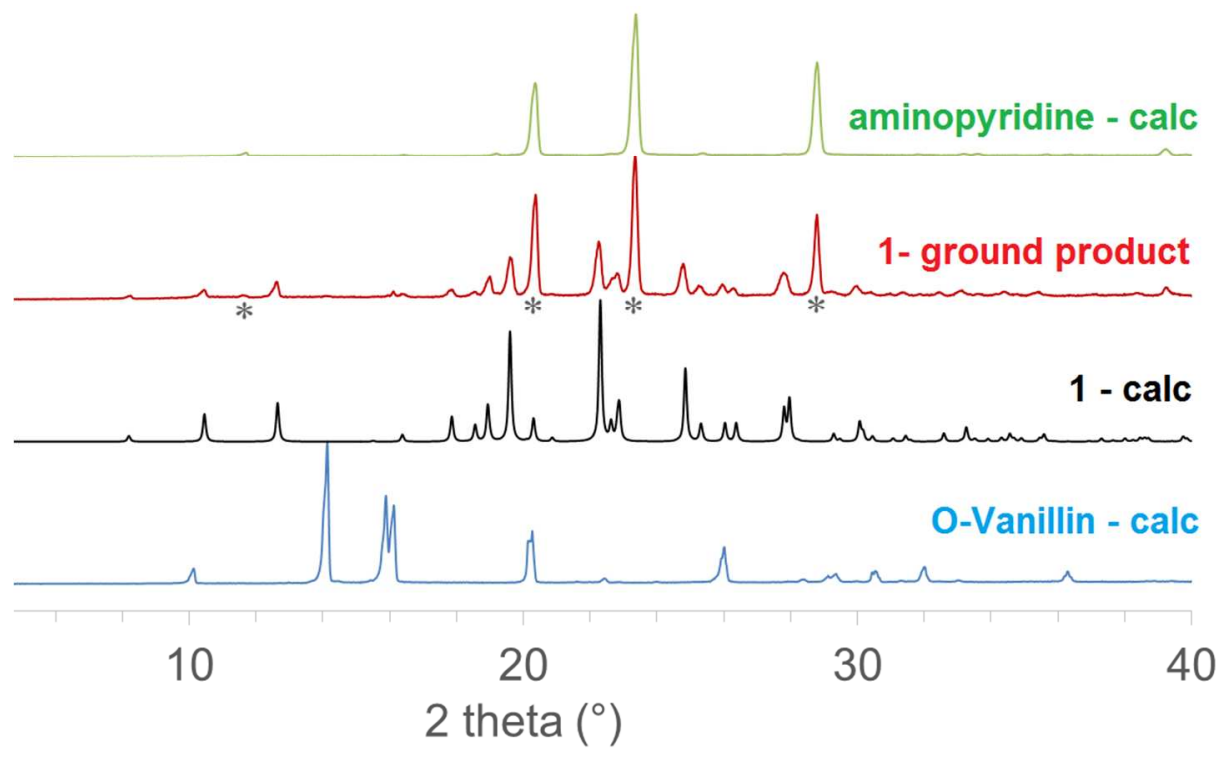

b)

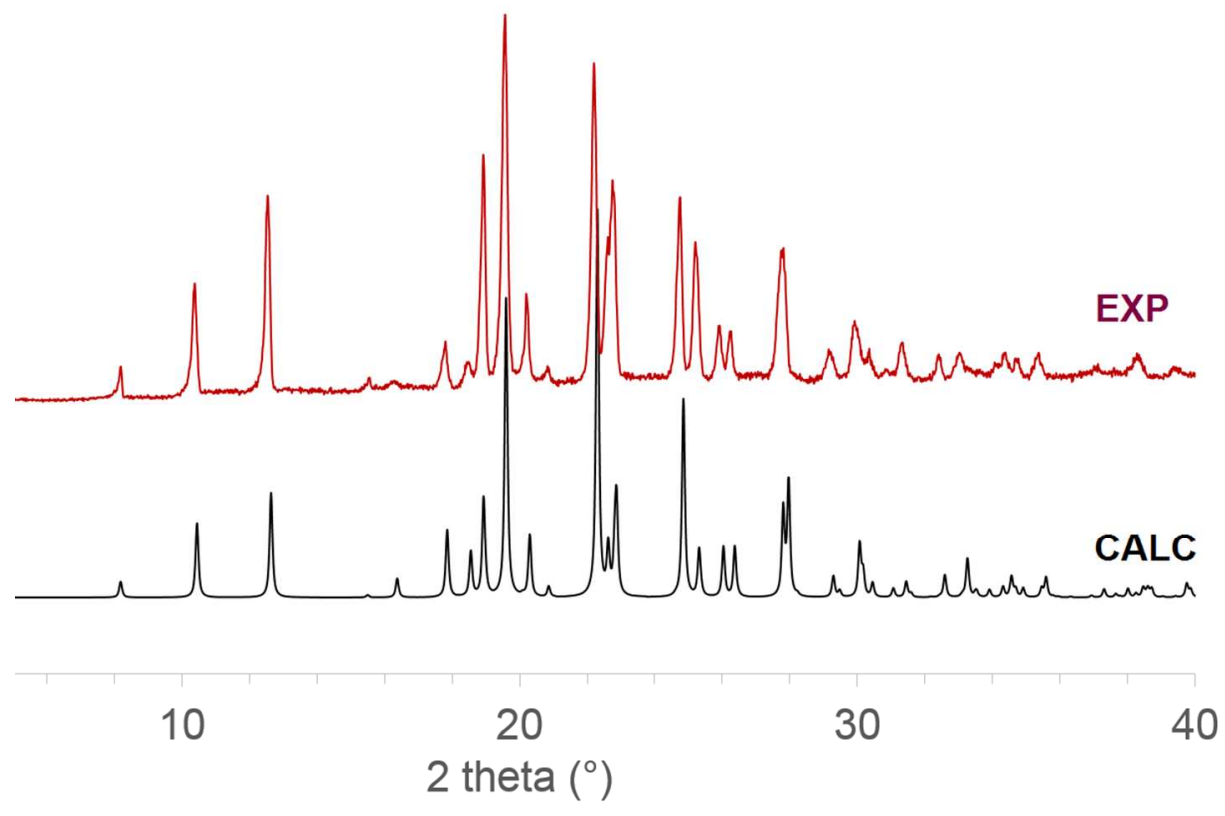

Figure S1. Powder X-ray diffraction measurement of the ground product of $\mathbf{1}$ before (a) and after (b) purification by recrystallization in EtOAc. Peaks indicated with black-stars are due to residual 3aminopyridine in the ground product taken directly ball milling. 
a)

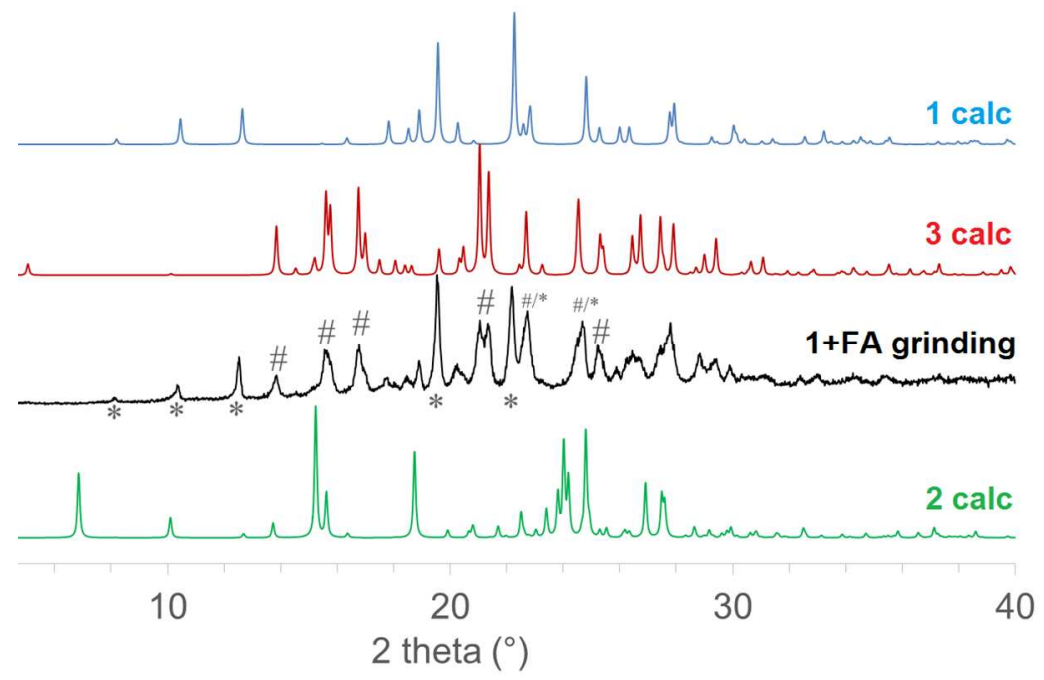

b)

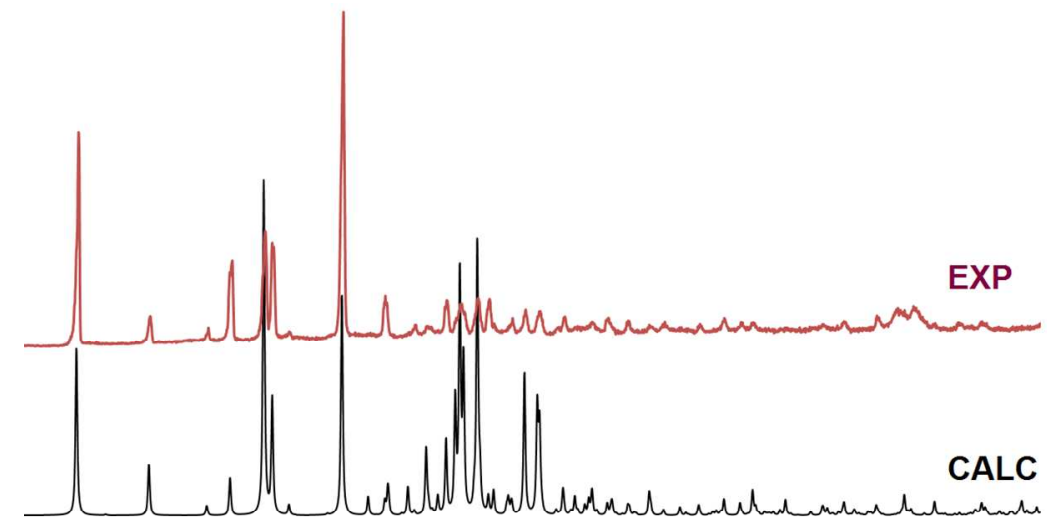

10

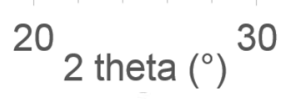

40

50

c)

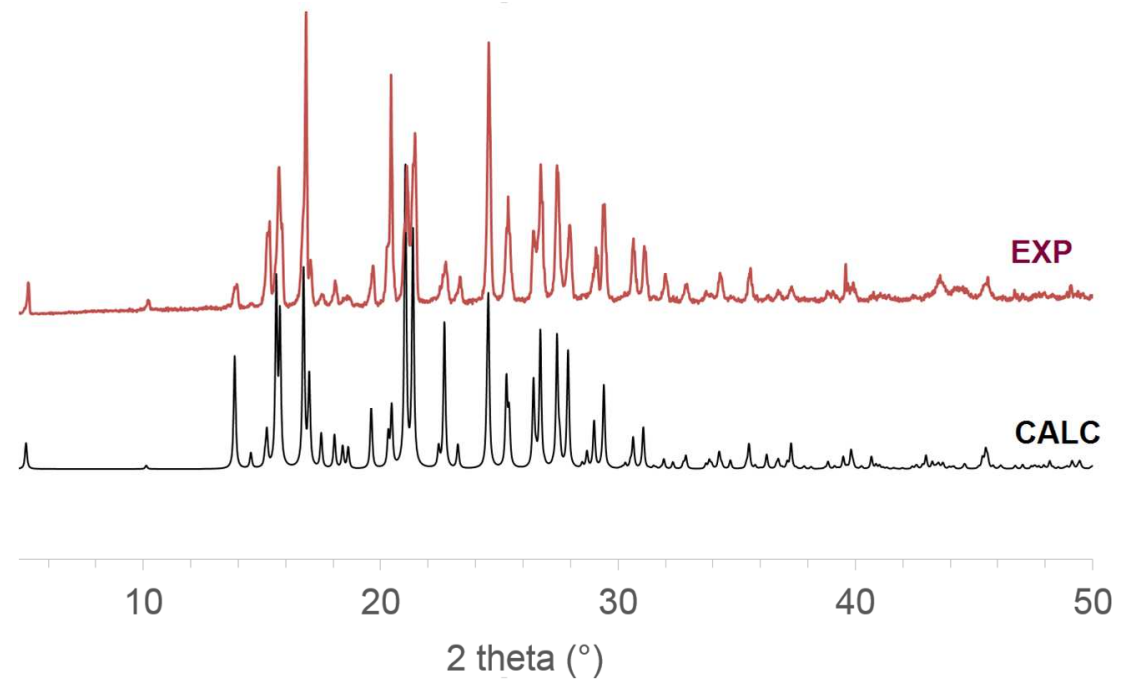

Figure S2. Powder X-ray diffraction measurement of the mechanosynthesis of $(\mathbf{1})_{2} \cdot \mathbf{F A}$ type cocrystals (a). PXRD of purified $\mathbf{2}$ (b) and $\mathbf{3}$ (c). Peaks indicated with black-stars are due to residual $\mathbf{1}$ in the ground product taken directly after ball milling. Peaks indicated with \# are attributed to formation of $\mathbf{3}$. 

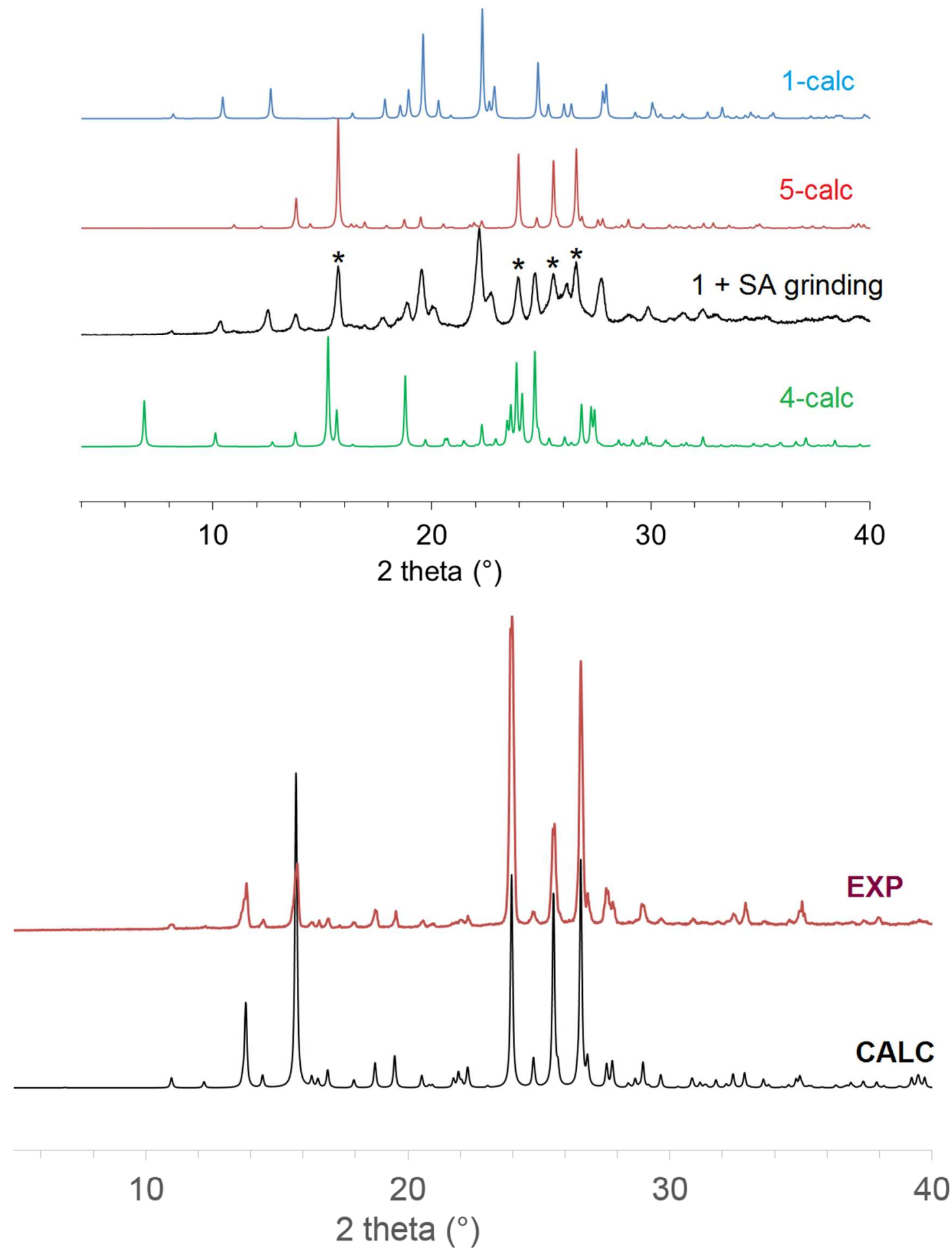

Figure S3. Powder X-ray diffraction measurement of the mechanosynthesis of $(\mathbf{1})_{2} \cdot \mathbf{S A}$ type cocrystals (a). PXRD of purified $\mathbf{5}$ (b). Peaks indicated with black-stars are due to formation of $\mathbf{5}$. 

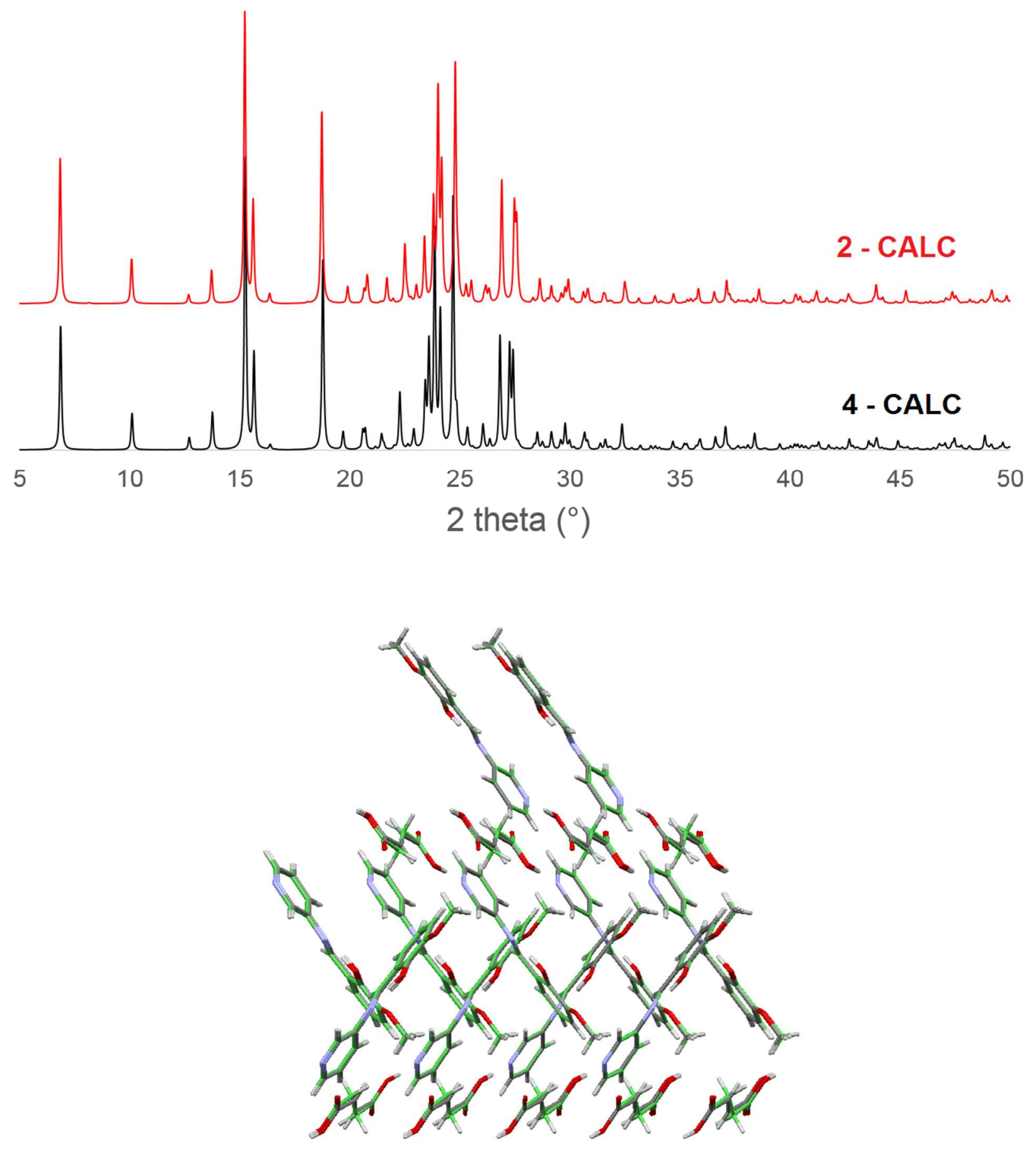

Figure S4. Calculated Powder X-ray diffractograms showing isostructurality between cocrystal 2 and cocrystal 4 (top) and perfect matching (bottom) between the two crystallographic structures ( 2 is represented in grey sticks while 4 is represented in green sticks, RMS $=0.098 \AA$ ). 
a)

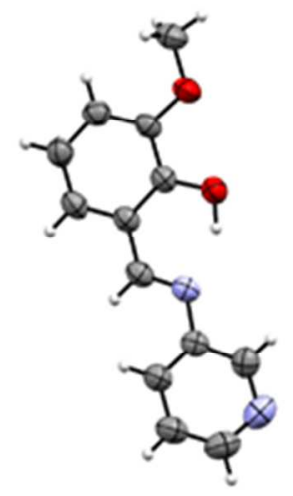
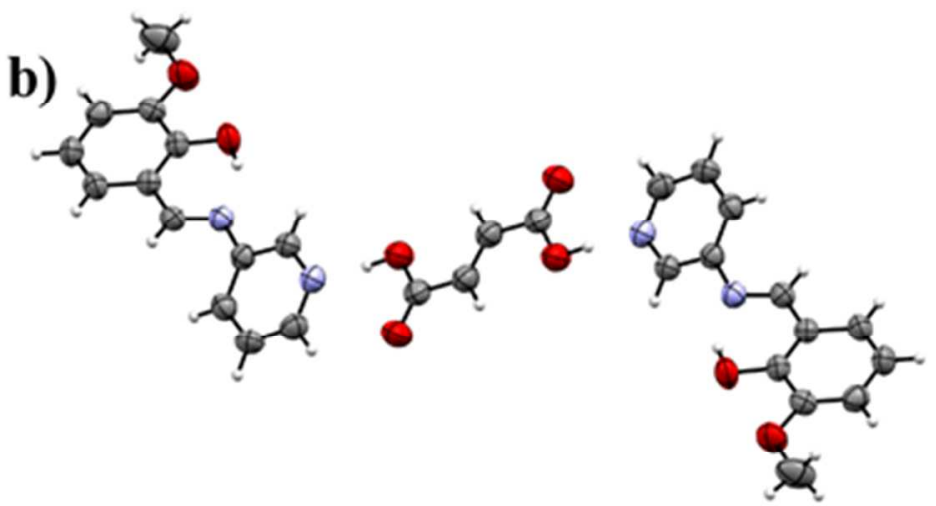

c)

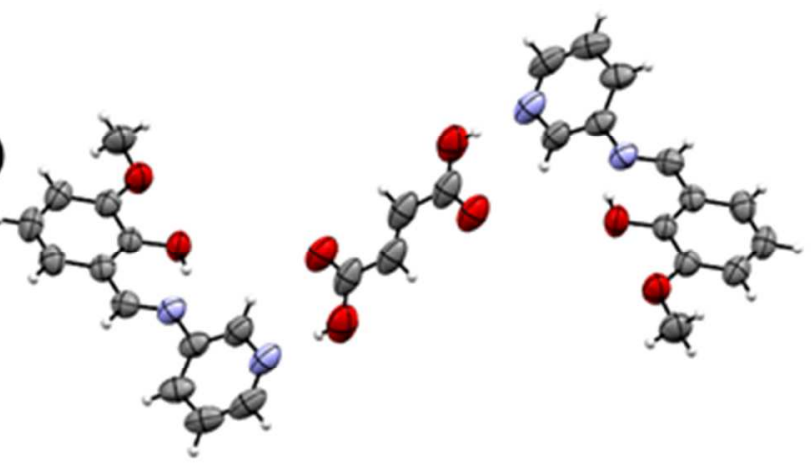

d)

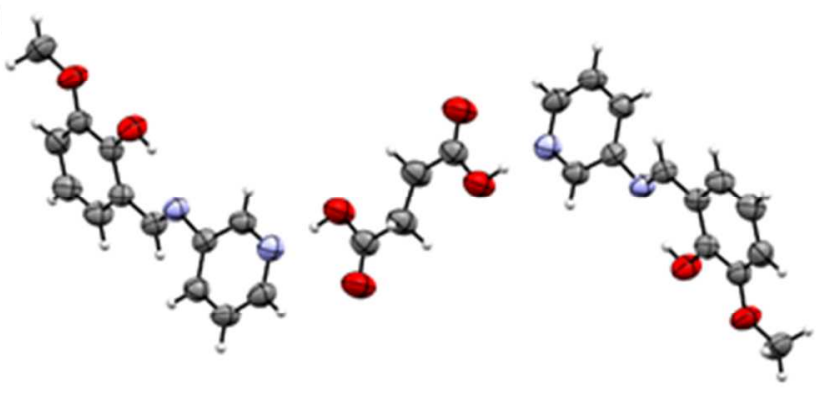

e)

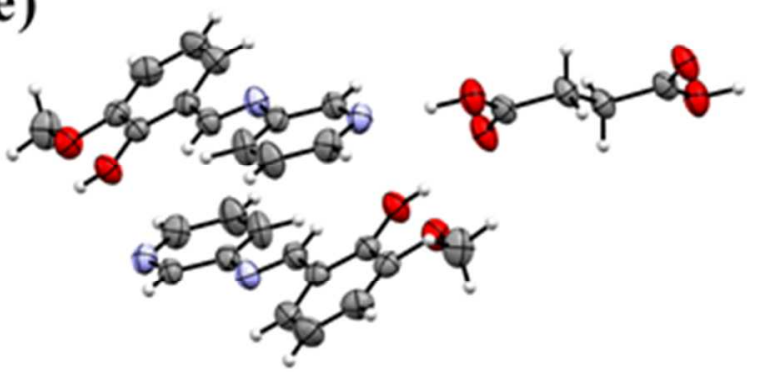

Figure S5. ORTEP diagrams (50\% probability ellipsoids) for $\mathbf{1}$ (a, 105K structure), 2 (b), 3 (c), 4 (d) and $5(\mathrm{e})$ 
a)

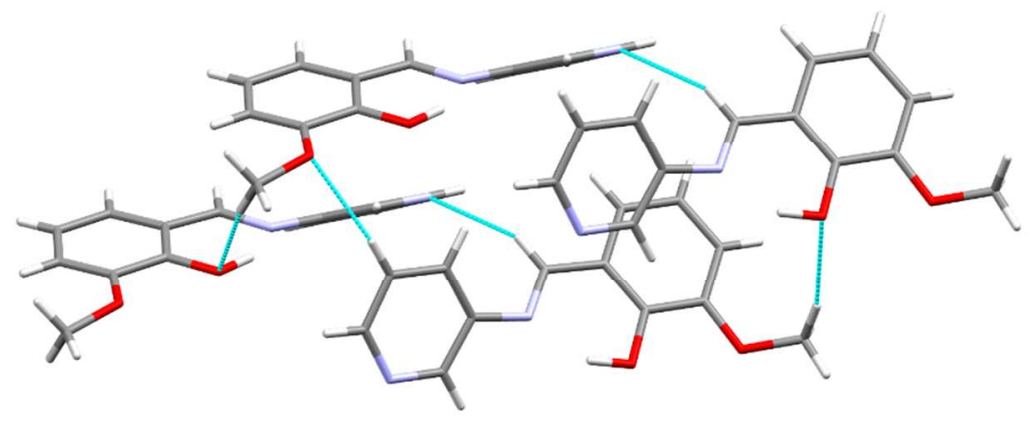

b)

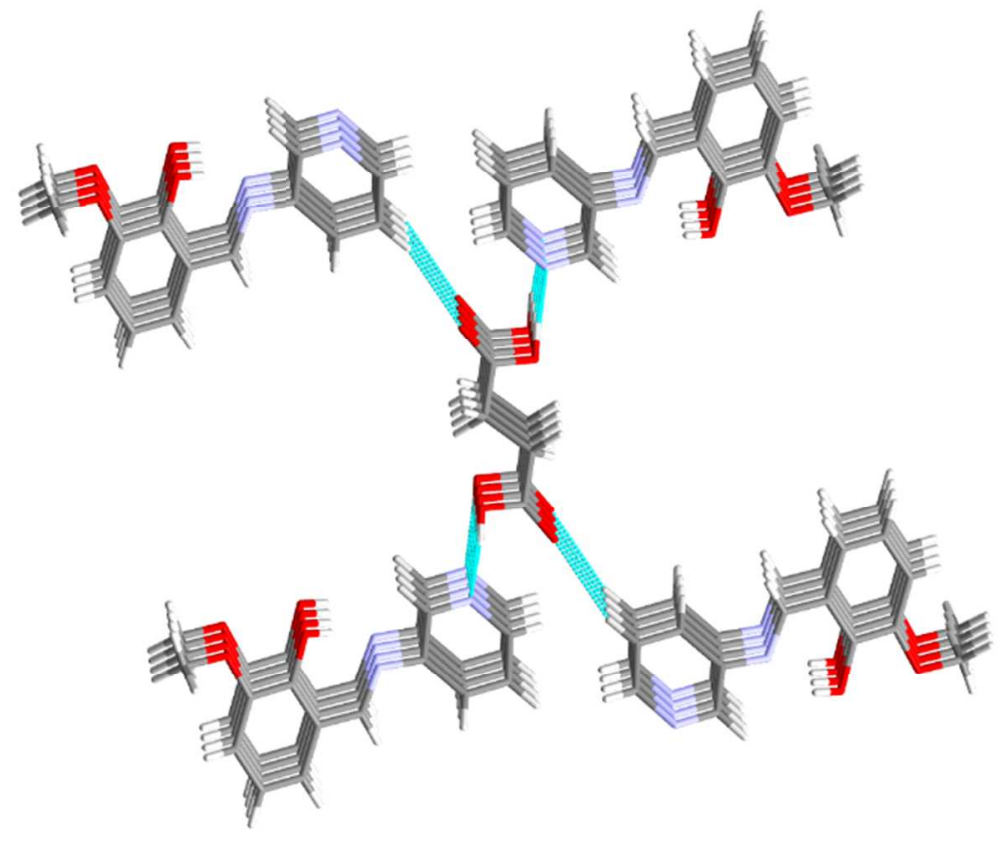

c)

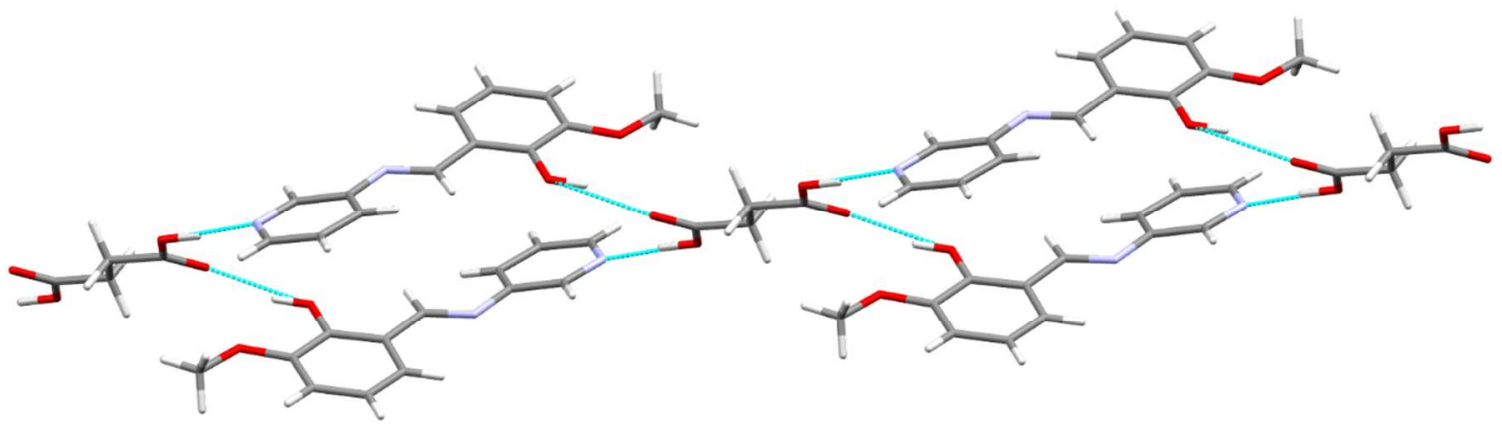

Figure S6. Crystal packing for 1 (a), 4 (b) and 5 (c) 

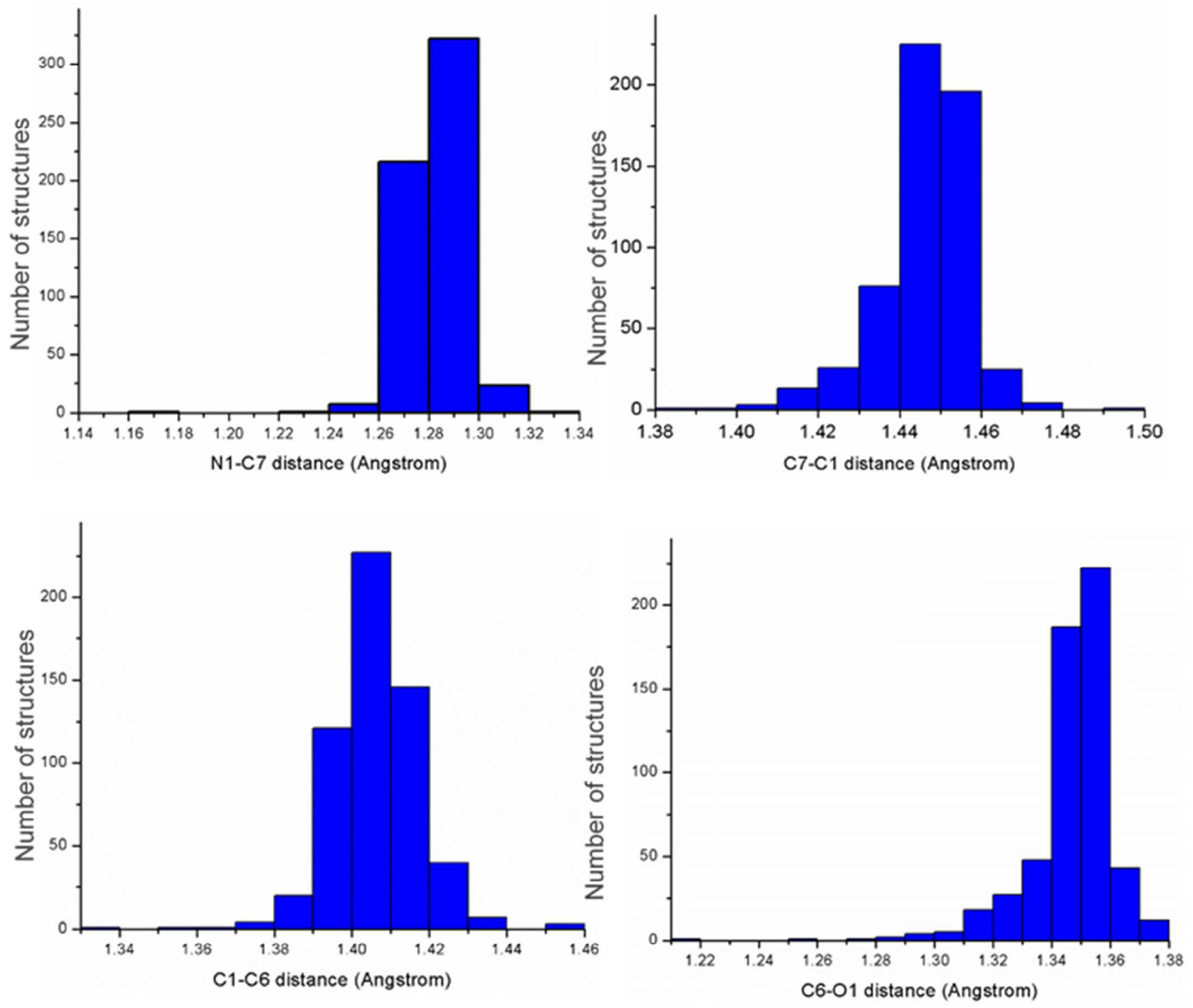

Figure S7. Histograms presenting the bond length in anils exhibiting their cis-enol form. Molecules have been retrieved from the CSD database (update January 2016). Only structures with $\mathrm{R} \%<5$ and with no disorder have been selected. 

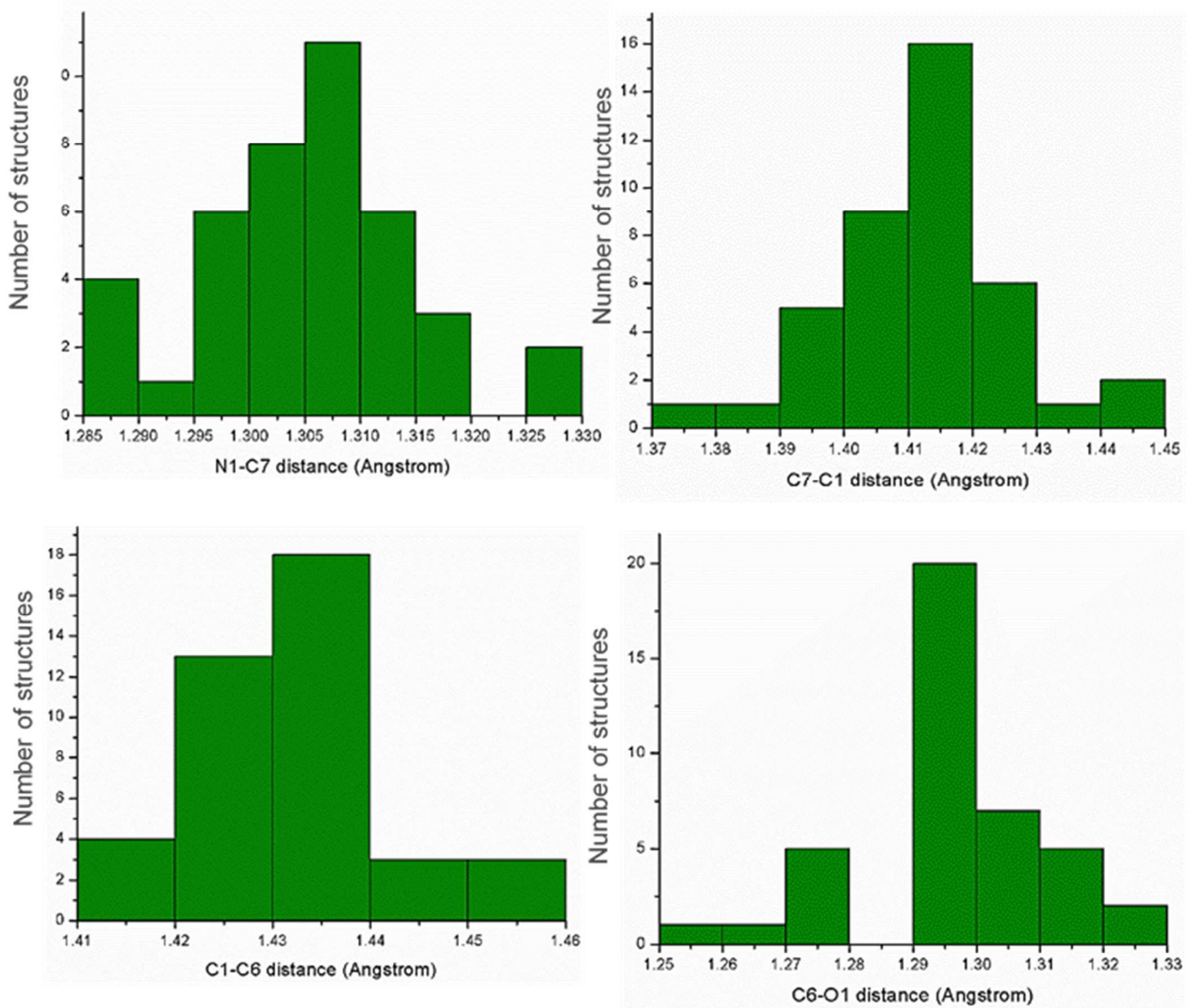

Figure S8. Histograms presenting the bond length in anils exhibiting their cis-keto form. Molecules have been retrieved from the CSD database (update January 2016). Only structures with $\mathrm{R} \%<5$ and with no disorder have been selected. 
a)
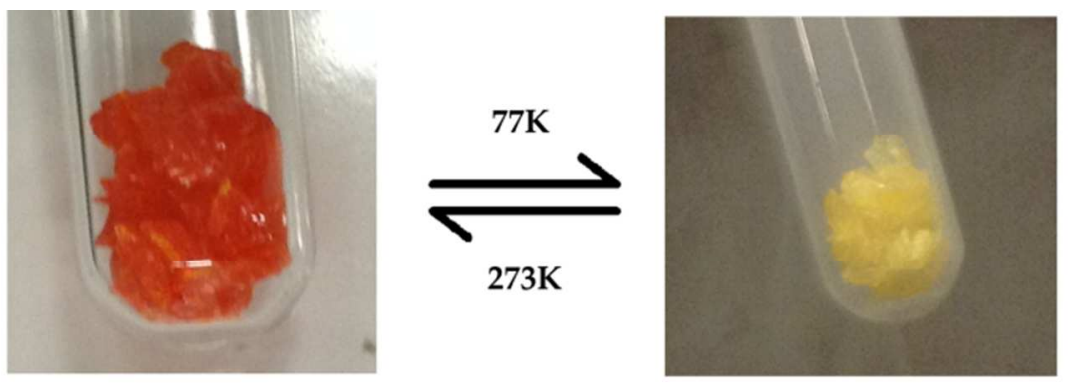

b)
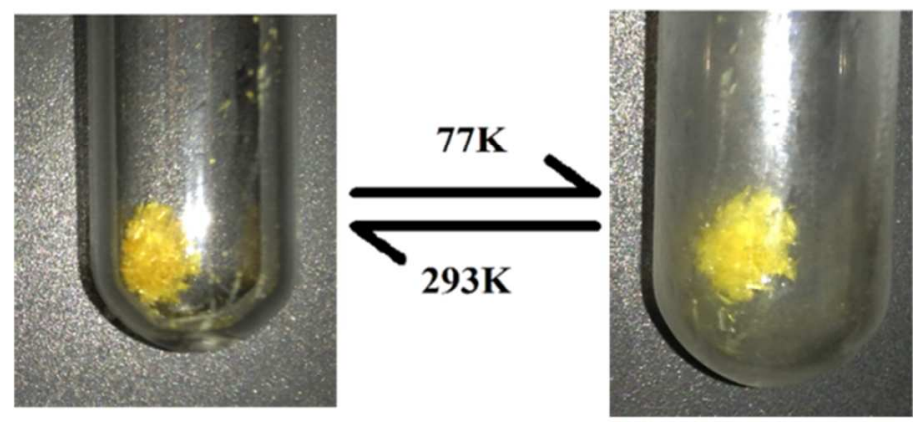

c)
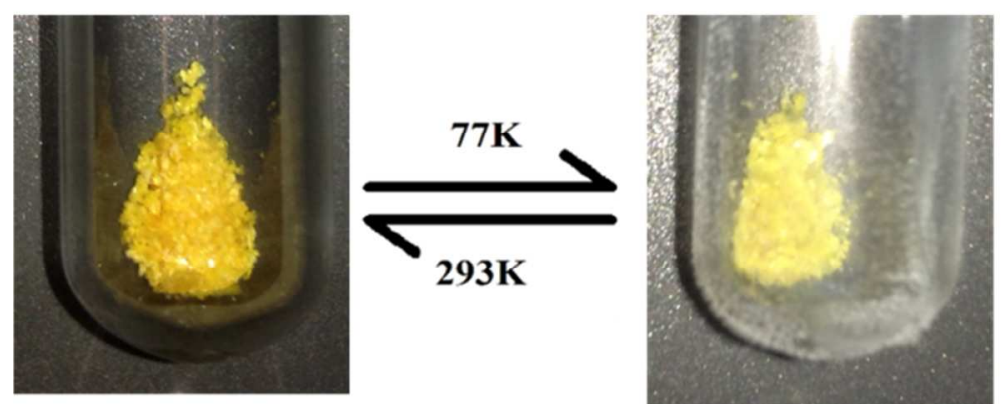

d)
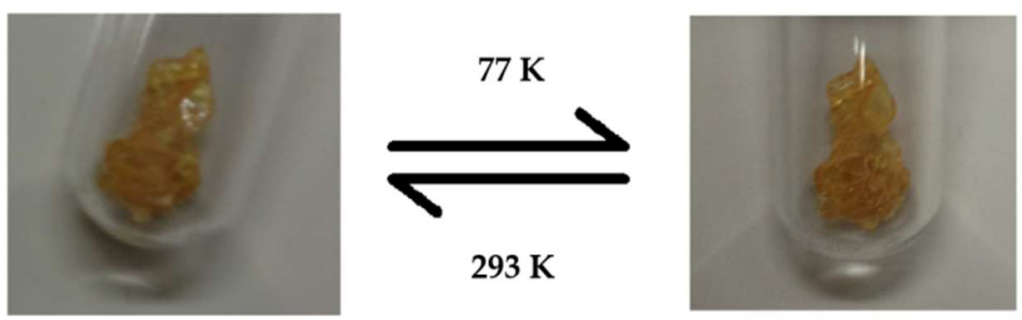

Figure S9. Liquid nitrogen exposure for 1-3 and 5. Crystals of one are strongly thermochromic, cocrystals 2-3 are slightly thermochromic. No color change observed for $\mathbf{5}$. 

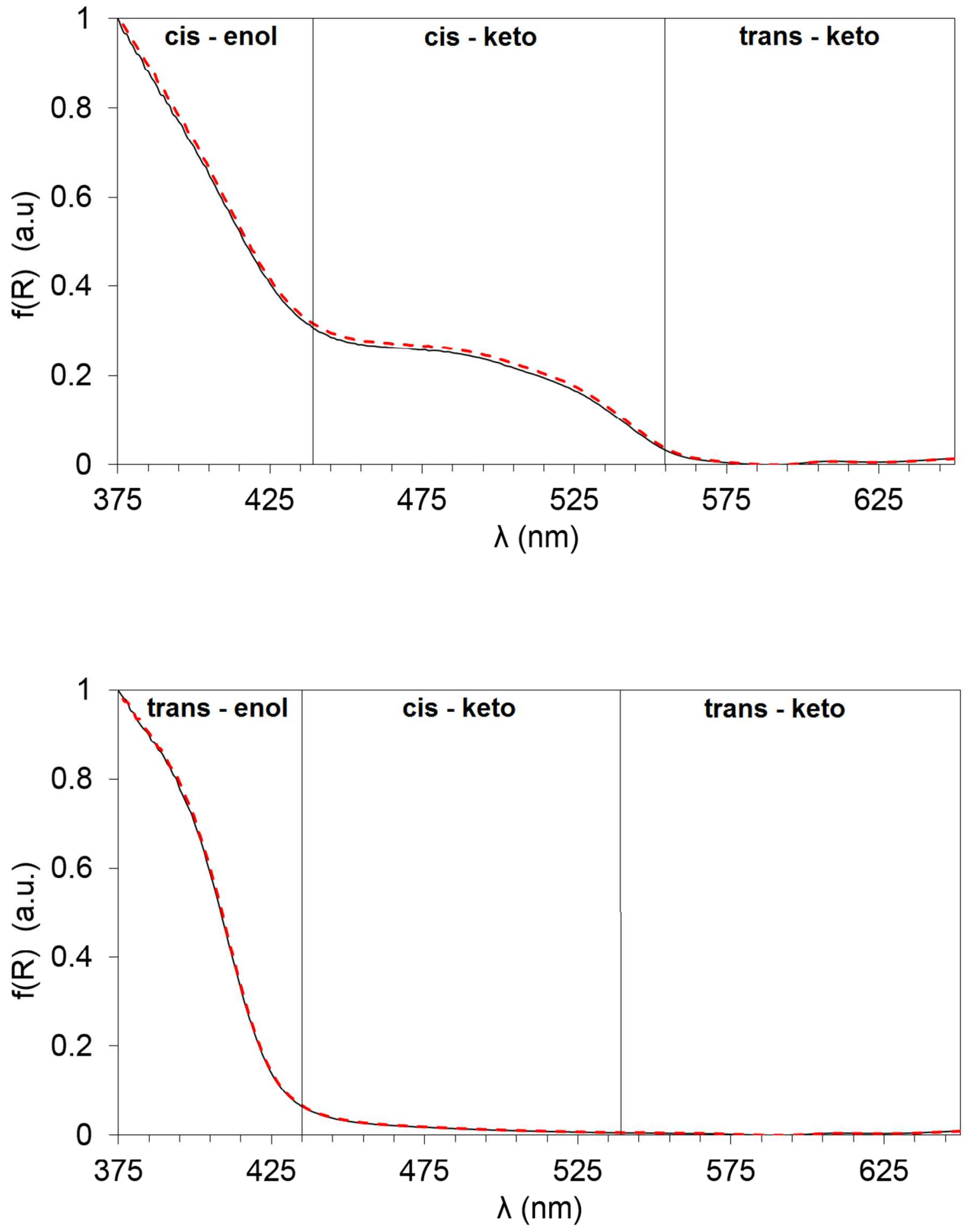

Figure S10. Kubelka-Munk spectra before (black solid line) and after (red dotted line) irradiation for 1 (top) and 5 (bottom) 


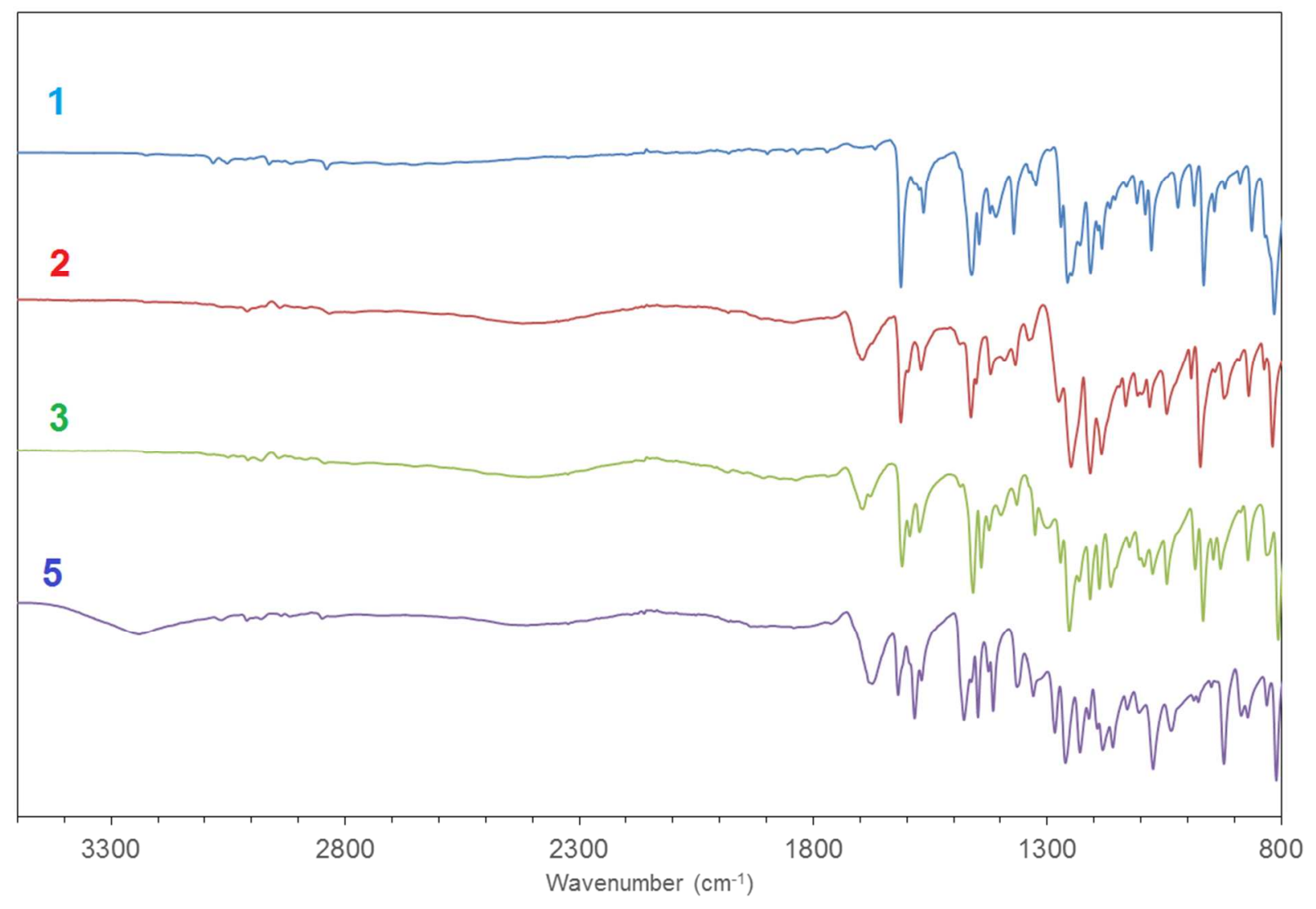

Figure S11. FTIR spectra of 1, 2, 3 and 5. 

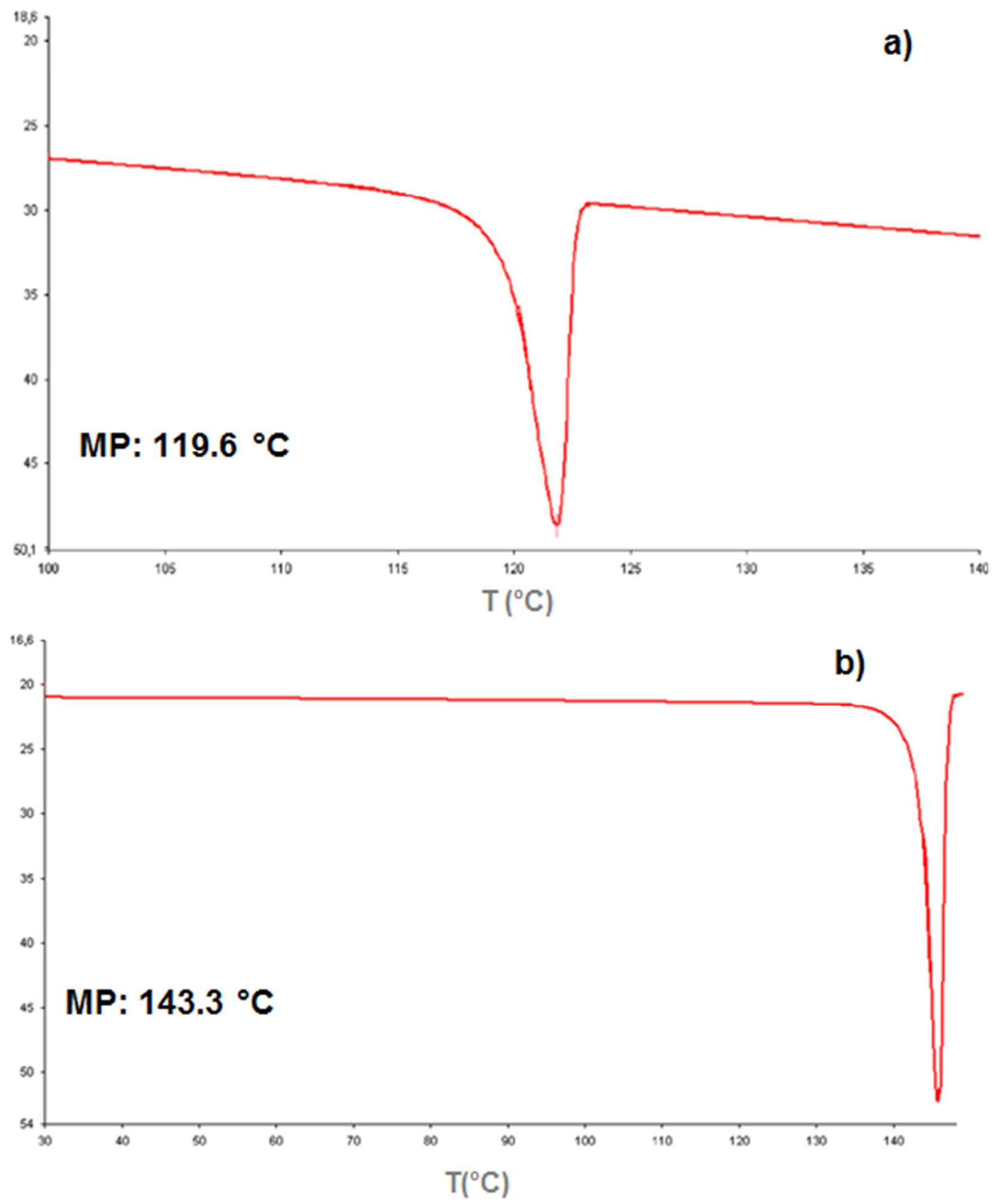

Figure S12. Melting point (onset), of crystals 1 and $\mathbf{2}$ measured by means of a Perkin Elmer DSC 7.0 calorimeter equipped with the PYRIS 1997-1998 program. 

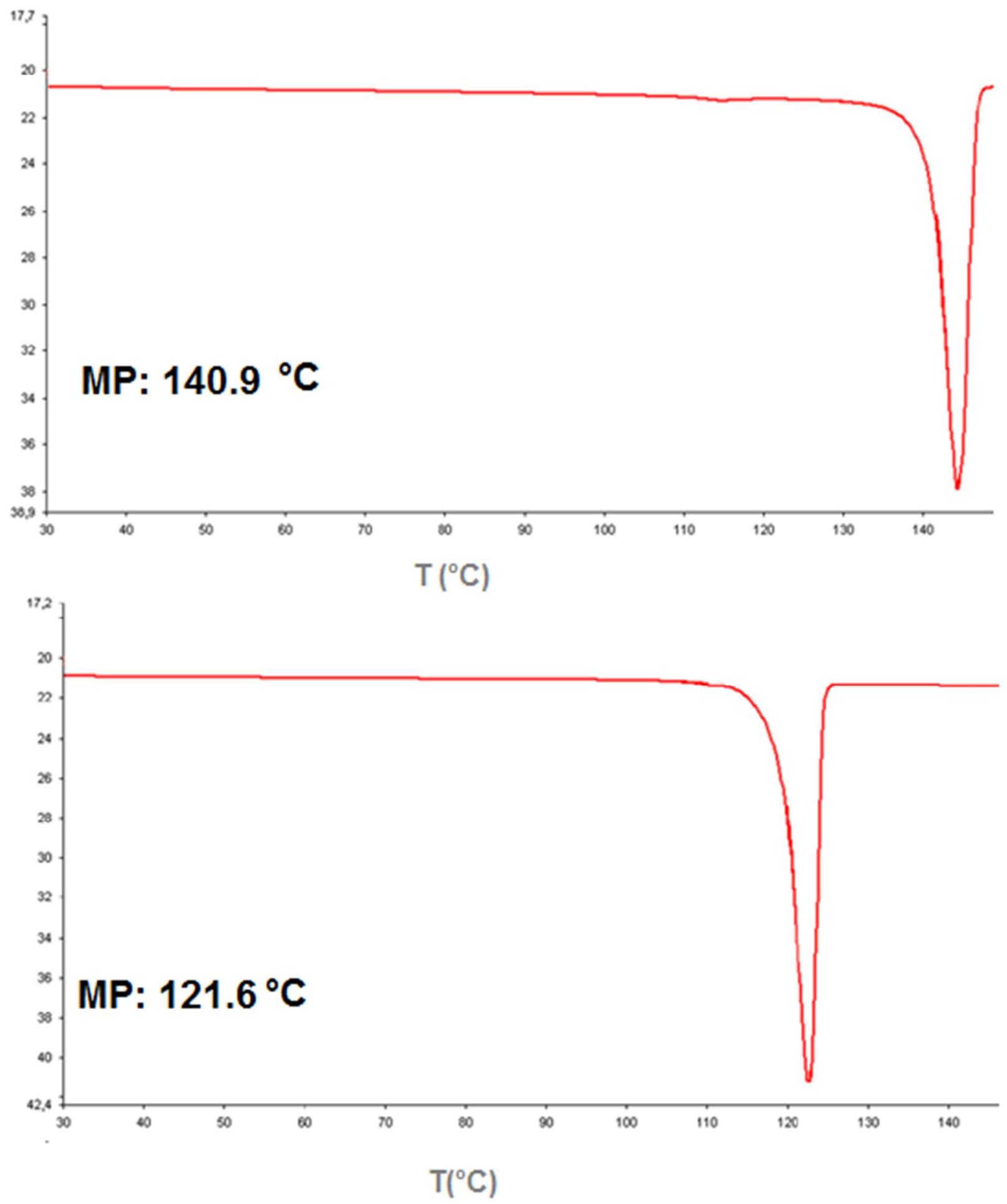

Figure S13. Melting point (onset) of cocrystals 3 (top) and 5 (bottom) measured by means of a Perkin Elmer DSC 7.0 calorimeter equipped with the PYRIS 1997-1998 program. Measurements have been performed in the range 30 to $150^{\circ} \mathrm{C}$ at a speed of $5^{\circ} \mathrm{C} / \mathrm{min}$ 
Table S1. Elemental analysis on crystals of $\mathbf{1 .}$

\begin{tabular}{ccc}
\hline Element & Calculated & Experimental \\
\hline $\mathbf{N}$ & $12.27 \%$ & $12.29 \%$ \\
$\mathbf{C}$ & $68.41 \%$ & $68.76 \%$ \\
$\mathbf{H}$ & $5.30 \%$ & $5.25 \%$ \\
$\mathbf{O}$ & $14.02 \%$ & $13.7 \%$ \\
& Empirical formula $\mathbf{C}_{\mathbf{1 3}} \mathbf{H}_{\mathbf{1 2}} \mathbf{N}_{\mathbf{2}} \mathbf{O}_{\mathbf{2}}$ & \\
\hline
\end{tabular}

Table S2. Room Temperature SCXRD measurements: crystallographic parameters

\begin{tabular}{|c|c|c|c|c|c|}
\hline & 1 & 2 & 3 & 4 & 5 \\
\hline Empirical & $\mathrm{C}_{13} \mathrm{H}_{12} \mathrm{~N}_{2} \mathrm{O}_{2}$ & $\mathrm{C}_{13} \mathrm{H}_{12} \mathrm{~N}_{2} \mathrm{O}_{2}$ & $\mathrm{C}_{13} \mathrm{H}_{12} \mathrm{~N}_{2} \mathrm{O}_{2}$ & $\mathrm{C}_{13} \mathrm{H}_{12} \mathrm{~N}_{2} \mathrm{O}_{2}$ & $\mathrm{C}_{13} \mathrm{H}_{12} \mathrm{~N}_{2} \mathrm{O}_{2}$ \\
\hline formula & & $0.5\left(\mathrm{C}_{4} \mathrm{H}_{4} \mathrm{O}_{4}\right)$ & $0.5\left(\mathrm{C}_{4} \mathrm{H}_{4} \mathrm{O}_{4}\right)$ & $0.5\left(\mathrm{C}_{4} \mathrm{H}_{6} \mathrm{O}_{4}\right)$ & $0.5\left(\mathrm{C}_{4} \mathrm{H}_{6} \mathrm{O}_{4}\right)$ \\
\hline Fw (g/mol) & 228.25 & 286.28 & 286.28 & 287,29 & 287,29 \\
\hline Crystal system & Orthorhombic & Monoclinic & Monoclinic & Monoclinic & Monoclinic \\
\hline Space group & $\mathrm{P} 2{ }_{1} 2_{1} 2_{1}$ & $\mathrm{P} 2{ }_{1} / \mathrm{n}$ & $\mathrm{P} 2{ }_{1} / \mathrm{n}$ & $\mathrm{P} 2{ }_{1} / \mathrm{n}$ & $\mathrm{I} 2 / \mathrm{a}$ \\
\hline a $(\AA)$ & $5.6190(4)$ & $4.5344(1)$ & $6.2578(2)$ & $4.5815(2)$ & $16.2457(15)$ \\
\hline b $(\AA)$ & $9.2825(7)$ & $25.7895(7)$ & $34.9486(11)$ & $25.7191(6)$ & $6.6244(4)$ \\
\hline c $(\AA)$ & $21.7490(14)$ & $11.9533(3)$ & $6.8516(3)$ & $11.9326(6)$ & $25.818(2)$ \\
\hline$\alpha\left(^{\circ}\right)$ & 90 & 90 & 90 & 90 & 90 \\
\hline$\beta\left(^{\circ}\right)$ & 90 & $93.524(2)$ & $108.390(4)$ & $93.106(4)$ & $97.188(7)$ \\
\hline$\gamma\left({ }^{\circ}\right)$ & 90 & 90 & 90 & 90 & 90 \\
\hline $\mathbf{V}\left(\AA^{3}\right)$ & $1134.39(14)$ & $1395.18(6)$ & $1421.93(10)$ & $1403.98(10)$ & $2756.7(4)$ \\
\hline $\mathbf{Z}$ & 4 & 4 & 4 & 4 & 8 \\
\hline Crystal size & $0.20 \times 0.40 \times$ & $0.12 \times 0.20 \times$ & $0.10 \times 0.1 \times$ & $0.06 \times 0.26 \times 0.5$ & $0.20 \times 0.30 \times$ \\
\hline$(\mathbf{m m})$ & 0.54 & 0.27 & 0.30 & & 0.50 \\
\hline $\mathbf{T}(\mathbf{K})$ & 293 & 293 & 293 & 293 & 293 \\
\hline Radiation (Å) & MoKa: 0.71073 & $\mathrm{CuK} \alpha: 1.54184$ & CuKa:1.54184 & $\mathrm{CuK \alpha} \alpha 1.54184$ & MoK $\alpha: 0.71073$ \\
\hline Nref, Npar & 1983,160 & 2462,203 & 2508,203 & 2474,207 & 2432,207 \\
\hline $\mathrm{R}[\mathrm{I}>\mathbf{2 \sigma}(\mathrm{I})]$ & $0.0370,1.10$ & $0.0458,1.30$ & $0.0417,1.27$ & $0.0411,1.04$ & $0.0375,1.00$ \\
\hline GoF， wR $\mathbf{R}^{2}$ & 0.0809 & 0.1289 & 0.1351 & 0.1175 & 0.0987 \\
\hline
\end{tabular}


Table S3. Selected geometries for H-bonds

\begin{tabular}{lllll}
\hline $\begin{array}{l}\text { Crystal } \\
\text { structure }\end{array}$ & $\mathbf{D}-\mathbf{H} \cdots \mathbf{A}$ & $\mathbf{H} \cdots \mathbf{A}(\AA)$ & $\mathbf{D} \cdots \mathbf{A}(\AA)$ & Angle $\left(^{\circ}\right)$ \\
\hline $\mathbf{1}$ & & & & \\
$\mathbf{2}$ & $\mathrm{O} 1-\mathrm{H} 1 \mathrm{O} \cdots \mathrm{N} 1$ & $1.74(3)$ & $2.611(2)$ & $151(3)$ \\
& $\mathrm{O} 1-\mathrm{H} 10 \cdots \mathrm{N} 1$ & $1.82(4)$ & $2.648(3)$ & $152(3)$ \\
$\mathbf{3}$ & $\mathrm{O} 3-\mathrm{H} 2 \mathrm{O} \cdots \mathrm{N} 2$ & $1.67(4)$ & $2.641(3)$ & $175(3)$ \\
& $\mathrm{O} 1-\mathrm{H} 1 \mathrm{O} \cdots \mathrm{N} 1$ & $1.69(3)$ & $2.582(3)$ & $153(3)$ \\
$\mathbf{4}$ & $\mathrm{O} 3-\mathrm{H} 2 \mathrm{O} \cdots \mathrm{N} 2$ & $1.81(3)$ & $2.622(3)$ & $176(3)$ \\
& $\mathrm{O} 1-\mathrm{H} 1 \mathrm{O} \cdots \mathrm{N} 1$ & $1.87(2)$ & $2.647(2)$ & $150(2)$ \\
$\mathbf{5}$ & $\mathrm{O} 3-\mathrm{H} 2 \mathrm{O} \cdots \mathrm{N} 2$ & $1.68(2)$ & $2.656(2)$ & $173(2)$ \\
& $\mathrm{O} 1-\mathrm{H} 1 \mathrm{O} \cdots \mathrm{O} 4$ & $1.95(2)$ & $2.780(2)$ & $155.9(2)$ \\
& $\mathrm{O} 3-\mathrm{H} 2 \mathrm{O} \cdots \mathrm{N} 2$ & $1.67(2)$ & $2.652(2)$ & $174(2)$ \\
\hline
\end{tabular}

Table S4. Selected geometries for carboxyl group in FA and SA.

\begin{tabular}{llll}
\hline $\begin{array}{l}\text { Crystal } \\
\text { structure }\end{array}$ & $\mathbf{C 1 4 - O 3}(\AA)$ & $\mathbf{C 1 4 - 0 4}(\AA)$ & Salt/Cocrystal \\
\hline $\mathbf{2}$ & $1.308(3)$ & $1.206(3)$ & Cocrystal \\
$\mathbf{3}$ & $1.302(4)$ & $1.220(3)$ & Cocrystal \\
$\mathbf{4}$ & $1.316(2)$ & $1.201(2)$ & Cocrystal \\
$\mathbf{5}$ & $1.312(2)$ & $1.218(2)$ & Cocrystal \\
\hline
\end{tabular}


Table S5. Bond lengths of the six-membered pseudocycle in anils under the enol form (415 retrieved structures; 530 total molecules). Chemical diagram for search is provided along with the table.

\begin{tabular}{|c|c|c|c|c|c|c|}
\hline \multirow[b]{2}{*}{ NAME } & \multirow[b]{2}{*}{$\begin{array}{c}\text { Number } \\
\text { of } \\
\text { molecules }\end{array}$} & \multirow[b]{2}{*}{$\begin{array}{c}\text { N1-C7 } \\
(\AA)\end{array}$} & \multirow[b]{2}{*}{$\begin{array}{c}\text { C7-C1 } \\
(\AA)\end{array}$} & \multicolumn{2}{|c|}{ Query for ENOL } & \multirow[b]{2}{*}{$\begin{array}{c}\text { O1-H1O } \\
(\AA)\end{array}$} \\
\hline & & & & $\begin{array}{c}\text { C1-C6 } \\
(\AA)\end{array}$ & $\begin{array}{c}\text { C6-01 } \\
(\AA)\end{array}$ & \\
\hline AFIKEH01 & 1 & 1.283 & 1.447 & 1.408 & 1.357 & 1.021 \\
\hline CORCOD & 1 & 1.286 & 1.458 & 1.416 & 1.335 & 0.815 \\
\hline EGUTAD & 1 & 1.277 & 1.457 & 1.402 & 1.347 & 0.82 \\
\hline EGUTEH & 1 & 1.292 & 1.45 & 1.414 & 1.337 & 0.84 \\
\hline GOKPAZ & 1 & 1.282 & 1.455 & 1.384 & 1.35 & 0.891 \\
\hline KOGFUJ & 1 & 1.291 & 1.438 & 1.404 & 1.327 & 1.018 \\
\hline KOGQUU & 1 & 1.259 & 1.45 & 1.412 & 1.338 & 0.82 \\
\hline KOGQUU & 2 & 1.277 & 1.456 & 1.403 & 1.335 & 0.821 \\
\hline MONFIG & 1 & 1.281 & 1.449 & 1.42 & 1.337 & 0.962 \\
\hline MONFUS & 1 & 1.28 & 1.453 & 1.414 & 1.324 & 0.96 \\
\hline TOMHOU & 1 & 1.282 & 1.449 & 1.396 & 1.358 & 0.921 \\
\hline TOQFAI & 1 & 1.281 & 1.447 & 1.386 & 1.354 & 0.82 \\
\hline TOQFAI & 2 & 1.279 & 1.445 & 1.393 & 1.352 & 0.819 \\
\hline TOQFEM & 1 & 1.283 & 1.441 & 1.389 & 1.348 & 0.815 \\
\hline TOQFEM & 2 & 1.275 & 1.438 & 1.388 & 1.352 & 0.834 \\
\hline TOQFEM & 3 & 1.286 & 1.447 & 1.385 & 1.351 & 0.924 \\
\hline TOQFEM & 4 & 1.28 & 1.445 & 1.392 & 1.349 & 0.914 \\
\hline TOQHIS & 1 & 1.288 & 1.425 & 1.407 & 1.324 & 0.82 \\
\hline WEFHAR01 & 1 & 1.282 & 1.454 & 1.403 & 1.358 & 0.825 \\
\hline WOMVAX & 1 & 1.287 & 1.453 & 1.408 & 1.352 & 0.84 \\
\hline XOFQAM & 1 & 1.295 & 1.419 & 1.42 & 1.348 & 0.82 \\
\hline ZOHNOB & 1 & 1.289 & 1.438 & 1.412 & 1.358 & 0.959 \\
\hline ABUVOJ & 1 & 1.298 & 1.425 & 1.415 & 1.353 & 0.981 \\
\hline ADIMEH & 1 & 1.286 & 1.445 & 1.411 & 1.355 & 0.946 \\
\hline AFILAE & 1 & 1.284 & 1.448 & 1.397 & 1.36 & 0.842 \\
\hline AGEGUQ & 1 & 1.266 & 1.453 & 1.411 & 1.327 & 0.82 \\
\hline AJETOZ & 1 & 1.279 & 1.438 & 1.402 & 1.351 & 0.82 \\
\hline
\end{tabular}




\begin{tabular}{|c|c|c|c|c|c|c|}
\hline AJETUF & 1 & 1.274 & 1.448 & 1.398 & 1.355 & 0.866 \\
\hline AJEYUK & 1 & 1.287 & 1.445 & 1.414 & 1.355 & 0.841 \\
\hline AJITOD & 1 & 1.282 & 1.437 & 1.401 & 1.346 & 0.852 \\
\hline AJITUJ & 1 & 1.279 & 1.439 & 1.403 & 1.345 & 0.856 \\
\hline ALOSEA & 1 & 1.273 & 1.441 & 1.414 & 1.346 & 0.841 \\
\hline AQAQOZ & 1 & 1.285 & 1.443 & 1.414 & 1.331 & 0.819 \\
\hline AVUYUM & 1 & 1.27 & 1.453 & 1.403 & 1.351 & 0.82 \\
\hline AWUSER & 1 & 1.278 & 1.453 & 1.405 & 1.347 & 0.88 \\
\hline AWUSER & 2 & 1.279 & 1.449 & 1.408 & 1.349 & 0.896 \\
\hline AWUSIV & 1 & 1.278 & 1.452 & 1.399 & 1.345 & 0.788 \\
\hline AYOQOV & 1 & 1.287 & 1.465 & 1.396 & 1.36 & 0.815 \\
\hline BALHUS & 1 & 1.281 & 1.454 & 1.408 & 1.366 & 0.976 \\
\hline BALHUS01 & 1 & 1.279 & 1.449 & 1.405 & 1.362 & 1.076 \\
\hline BANGOM01 & 1 & 1.287 & 1.45 & 1.416 & 1.352 & 0.835 \\
\hline BANYIZ & 1 & 1.299 & 1.432 & 1.422 & 1.353 & 0.886 \\
\hline BIKLEO & 1 & 1.285 & 1.451 & 1.408 & 1.353 & 0.903 \\
\hline BSALAP01 & 1 & 1.281 & 1.467 & 1.399 & 1.346 & 0.735 \\
\hline BUJVAE & 1 & 1.283 & 1.45 & 1.416 & 1.345 & 0.79 \\
\hline BUJVEI & 1 & 1.283 & 1.456 & 1.409 & 1.346 & 0.825 \\
\hline BUJVEI & 2 & 1.282 & 1.458 & 1.409 & 1.343 & 0.904 \\
\hline BUJVEI & 3 & 1.283 & 1.457 & 1.413 & 1.347 & 0.831 \\
\hline CAQYAW & 1 & 1.287 & 1.439 & 1.404 & 1.337 & 0.889 \\
\hline CAQYAW01 & 1 & 1.277 & 1.445 & 1.402 & 1.343 & 1.005 \\
\hline CAVQAR & 1 & 1.273 & 1.443 & 1.405 & 1.353 & 0.943 \\
\hline CAVQIZ & 1 & 1.28 & 1.447 & 1.411 & 1.352 & 0.832 \\
\hline CAVQOF & 1 & 1.286 & 1.445 & 1.413 & 1.351 & 0.921 \\
\hline CEBKEZ & 1 & 1.286 & 1.435 & 1.417 & 1.345 & 1.009 \\
\hline CEBKEZ01 & 1 & 1.305 & 1.452 & 1.398 & 1.343 & 0.862 \\
\hline CEBKEZ02 & 1 & 1.287 & 1.455 & 1.411 & 1.353 & 0.97 \\
\hline CEGHED & 1 & 1.283 & 1.443 & 1.401 & 1.356 & 0.82 \\
\hline CEGVIV & 1 & 1.292 & 1.429 & 1.406 & 1.328 & 0.819 \\
\hline CELPEP & 1 & 1.279 & 1.449 & 1.411 & 1.327 & 0.82 \\
\hline CELPEP & 2 & 1.286 & 1.434 & 1.419 & 1.312 & 0.82 \\
\hline CHLSAN & 1 & 1.281 & 1.456 & 1.396 & 1.371 & 1.155 \\
\hline CICTOY & 1 & 1.285 & 1.447 & 1.397 & 1.354 & 0.906 \\
\hline CIKPOC & 1 & 1.266 & 1.458 & 1.409 & 1.333 & 0.82 \\
\hline CIKPOC & 2 & 1.28 & 1.458 & 1.409 & 1.337 & 0.821 \\
\hline CIRDEO & 1 & 1.276 & 1.447 & 1.398 & 1.342 & 0.82 \\
\hline CIWXIR & 1 & 1.281 & 1.445 & 1.399 & 1.35 & 0.943 \\
\hline CIXNOO & 1 & 1.295 & 1.419 & 1.42 & 1.306 & 0.82 \\
\hline CIXNOO & 2 & 1.293 & 1.43 & 1.409 & 1.316 & 0.82 \\
\hline CMXSAP & 1 & 1.279 & 1.446 & 1.428 & 1.342 & 0.852 \\
\hline COQSOR & 1 & 1.286 & 1.442 & 1.407 & 1.359 & 0.867 \\
\hline COQSOR & 2 & 1.282 & 1.449 & 1.409 & 1.363 & 0.881 \\
\hline CSALAN02 & 1 & 1.291 & 1.457 & 1.412 & 1.35 & 0.91 \\
\hline CUCZUW & 1 & 1.278 & 1.447 & 1.395 & 1.355 & 0.869 \\
\hline
\end{tabular}




\begin{tabular}{|c|c|c|c|c|c|c|}
\hline CUDDEL & 1 & 1.276 & 1.454 & 1.396 & 1.349 & 0.82 \\
\hline CULPOO & 1 & 1.294 & 1.454 & 1.4 & 1.347 & 0.883 \\
\hline CULPOO01 & 1 & 1.286 & 1.454 & 1.408 & 1.354 & 0.89 \\
\hline DASPAO & 1 & 1.281 & 1.451 & 1.386 & 1.355 & 0.993 \\
\hline DASPAO01 & 1 & 1.266 & 1.457 & 1.371 & 1.349 & 0.864 \\
\hline DASPAO01 & 2 & 1.274 & 1.455 & 1.386 & 1.354 & 1.118 \\
\hline DAWFAJ & 1 & 1.29 & 1.439 & 1.419 & 1.35 & 1.01 \\
\hline DICLAD & 1 & 1.276 & 1.454 & 1.402 & 1.362 & 0.82 \\
\hline DICLAD & 2 & 1.28 & 1.448 & 1.394 & 1.362 & 0.82 \\
\hline DIDRAL & 1 & 1.284 & 1.447 & 1.407 & 1.342 & 0.84 \\
\hline DIDRAL & 2 & 1.285 & 1.449 & 1.413 & 1.351 & 0.841 \\
\hline DOXRUE & 1 & 1.282 & 1.457 & 1.407 & 1.36 & 1.019 \\
\hline DOXRUE & 2 & 1.286 & 1.456 & 1.406 & 1.36 & 0.96 \\
\hline DOXSAL & 1 & 1.28 & 1.451 & 1.409 & 1.352 & 0.977 \\
\hline DOXSAL & 2 & 1.255 & 1.467 & 1.399 & 1.352 & 0.977 \\
\hline DUBNAQ & 1 & 1.278 & 1.451 & 1.405 & 1.351 & 0.748 \\
\hline DUJJOI & 1 & 1.294 & 1.466 & 1.412 & 1.376 & 0.82 \\
\hline DUMXEP & 1 & 1.284 & 1.439 & 1.411 & 1.345 & 0.948 \\
\hline DUMXUF & 1 & 1.282 & 1.446 & 1.416 & 1.351 & 0.823 \\
\hline DUPGOL & 1 & 1.276 & 1.452 & 1.408 & 1.351 & 0.933 \\
\hline DUSZAT & 1 & 1.286 & 1.445 & 1.419 & 1.352 & 0.872 \\
\hline EBUXUV & 1 & 1.25 & 1.468 & 1.428 & 1.338 & 0.82 \\
\hline EBUXUV & 2 & 1.309 & 1.427 & 1.332 & 1.371 & 1.045 \\
\hline EDEQAG & 1 & 1.282 & 1.446 & 1.405 & 1.348 & 0.93 \\
\hline EDIDUR & 1 & 1.267 & 1.438 & 1.412 & 1.36 & 0.977 \\
\hline EDIDUR & 2 & 1.279 & 1.452 & 1.411 & 1.361 & 1.027 \\
\hline EGUTAC & 1 & 1.277 & 1.442 & 1.408 & 1.348 & 0.82 \\
\hline EGUTEG & 1 & 1.263 & 1.461 & 1.411 & 1.329 & 0.82 \\
\hline EHOLAP & 1 & 1.275 & 1.45 & 1.41 & 1.34 & 0.82 \\
\hline EHUHIZ & 1 & 1.281 & 1.449 & 1.415 & 1.325 & 0.821 \\
\hline EKOROM & 1 & 1.287 & 1.446 & 1.403 & 1.344 & 0.84 \\
\hline EKUGIA & 1 & 1.271 & 1.456 & 1.417 & 1.335 & 0.813 \\
\hline ETEYUX & 1 & 1.284 & 1.435 & 1.402 & 1.35 & 0.82 \\
\hline ETEYUX & 2 & 1.278 & 1.441 & 1.393 & 1.355 & 0.821 \\
\hline EVIMEB & 1 & 1.271 & 1.46 & 1.398 & 1.347 & 0.995 \\
\hline EYEFOE & 1 & 1.285 & 1.443 & 1.41 & 1.349 & 0.872 \\
\hline FADBUI & 1 & 1.295 & 1.438 & 1.409 & 1.333 & 1.01 \\
\hline FAQHOU & 1 & 1.269 & 1.451 & 1.41 & 1.332 & 0.913 \\
\hline FASWIH & 1 & 1.282 & 1.46 & 1.407 & 1.353 & 0.82 \\
\hline FAXWOS & 1 & 1.278 & 1.452 & 1.413 & 1.359 & 0.82 \\
\hline FAXWOS & 2 & 1.271 & 1.457 & 1.406 & 1.35 & 0.821 \\
\hline FEDZOE & 1 & 1.279 & 1.442 & 1.403 & 1.348 & 0.82 \\
\hline FEVVEI & 1 & 1.249 & 1.435 & 1.351 & 1.327 & 0.839 \\
\hline FEVVEI & 2 & 1.283 & 1.439 & 1.383 & 1.359 & 0.84 \\
\hline FEVVEI & 3 & 1.273 & 1.435 & 1.402 & 1.346 & 0.839 \\
\hline FEVVEI & 4 & 1.273 & 1.448 & 1.388 & 1.338 & 0.84 \\
\hline
\end{tabular}




\begin{tabular}{|c|c|c|c|c|c|c|}
\hline FEXRIK & 1 & 1.265 & 1.456 & 1.395 & 1.377 & 0.993 \\
\hline FEXRIK & 2 & 1.28 & 1.445 & 1.41 & 1.316 & 0.993 \\
\hline FEXRIK01 & 1 & 1.284 & 1.447 & 1.403 & 1.351 & 0.923 \\
\hline FEXRIK01 & 2 & 1.279 & 1.446 & 1.397 & 1.35 & 0.934 \\
\hline FEXRIK01 & 3 & 1.283 & 1.449 & 1.398 & 1.348 & 0.925 \\
\hline FEXRIK01 & 4 & 1.272 & 1.451 & 1.388 & 1.358 & 1.088 \\
\hline FEXRIK02 & 1 & 1.279 & 1.449 & 1.4 & 1.348 & 0.923 \\
\hline FEXRIK02 & 2 & 1.277 & 1.45 & 1.396 & 1.347 & 0.929 \\
\hline FEXRIK02 & 3 & 1.279 & 1.446 & 1.395 & 1.348 & 0.914 \\
\hline FEXRIK02 & 4 & 1.274 & 1.444 & 1.392 & 1.353 & 0.999 \\
\hline FOCYEC & 1 & 1.268 & 1.454 & 1.402 & 1.354 & 0.598 \\
\hline FOCYOM & 1 & 1.276 & 1.438 & 1.413 & 1.348 & 0.974 \\
\hline FOJNEY & 1 & 1.27 & 1.438 & 1.372 & 1.344 & 0.82 \\
\hline FOJNEY & 2 & 1.279 & 1.447 & 1.388 & 1.349 & 0.82 \\
\hline FOVRUE02 & 1 & 1.296 & 1.449 & 1.403 & 1.341 & 0.82 \\
\hline FOVRUE03 & 1 & 1.294 & 1.441 & 1.407 & 1.333 & 0.819 \\
\hline FOWXOF & 1 & 1.275 & 1.429 & 1.416 & 1.328 & 0.82 \\
\hline FUHJOI & 1 & 1.336 & 1.43 & 1.45 & 1.372 & 0.821 \\
\hline FUHQUV & 1 & 1.283 & 1.44 & 1.412 & 1.351 & 0.926 \\
\hline GAWKEV & 1 & 1.277 & 1.429 & 1.4 & 1.354 & 0.821 \\
\hline GEHНEH & 1 & 1.27 & 1.449 & 1.396 & 1.336 & 0.942 \\
\hline GEJCII & 1 & 1.27 & 1.44 & 1.399 & 1.346 & 0.821 \\
\hline GEKZEC & 1 & 1.28 & 1.424 & 1.394 & 1.343 & 0.908 \\
\hline GETXEJ & 1 & 1.291 & 1.449 & 1.396 & 1.346 & 0.861 \\
\hline GETXEJ & 2 & 1.289 & 1.44 & 1.398 & 1.344 & 0.949 \\
\hline GIBZOG & 1 & 1.297 & 1.423 & 1.402 & 1.329 & 0.973 \\
\hline GISFOE & 1 & 1.273 & 1.44 & 1.399 & 1.354 & 0.82 \\
\hline GISFOE & 2 & 1.279 & 1.45 & 1.408 & 1.35 & 0.82 \\
\hline GOFLAP & 1 & 1.278 & 1.45 & 1.413 & 1.353 & 1.208 \\
\hline GONNUS & 1 & 1.282 & 1.446 & 1.412 & 1.349 & 1.141 \\
\hline GUSXIC & 1 & 1.278 & 1.455 & 1.408 & 1.349 & 0.841 \\
\hline GUVQOE & 1 & 1.275 & 1.452 & 1.403 & 1.348 & 0.859 \\
\hline HACZER & 1 & 1.273 & 1.46 & 1.407 & 1.34 & 0.91 \\
\hline HAKZEZ & 1 & 1.276 & 1.439 & 1.399 & 1.347 & 0.806 \\
\hline HALGUW & 1 & 1.285 & 1.429 & 1.394 & 1.357 & 0.997 \\
\hline HAMKIR & 1 & 1.288 & 1.433 & 1.408 & 1.346 & 0.98 \\
\hline HATLUK & 1 & 1.302 & 1.427 & 1.439 & 1.258 & 0.82 \\
\hline HEGQUH & 1 & 1.296 & 1.426 & 1.409 & 1.361 & 0.838 \\
\hline HEGQUH & 2 & 1.294 & 1.431 & 1.413 & 1.361 & 0.766 \\
\hline HEWFUM & 1 & 1.278 & 1.445 & 1.404 & 1.346 & 0.82 \\
\hline HEWGAT & 1 & 1.282 & 1.448 & 1.414 & 1.353 & 0.938 \\
\hline HEWGEX & 1 & 1.28 & 1.451 & 1.415 & 1.343 & 0.84 \\
\hline HEWGIB & 1 & 1.278 & 1.453 & 1.411 & 1.344 & 0.84 \\
\hline HICVEV & 1 & 1.289 & 1.412 & 1.408 & 1.297 & 0.82 \\
\hline HIDVEW & 1 & 1.273 & 1.433 & 1.414 & 1.348 & 0.82 \\
\hline HIRCIV & 1 & 1.276 & 1.451 & 1.396 & 1.355 & 0.812 \\
\hline
\end{tabular}




\begin{tabular}{|c|c|c|c|c|c|c|}
\hline HIVXUG & 1 & 1.284 & 1.452 & 1.402 & 1.35 & 0.96 \\
\hline HIVXUG & 2 & 1.287 & 1.451 & 1.4 & 1.347 & 0.959 \\
\hline HODDIO01 & 1 & 1.288 & 1.45 & 1.406 & 1.346 & 0.84 \\
\hline HODNUK & 1 & 1.278 & 1.447 & 1.399 & 1.357 & 0.803 \\
\hline HOFNEV & 1 & 1.29 & 1.445 & 1.407 & 1.334 & 0.946 \\
\hline HUDLAU & 1 & 1.268 & 1.447 & 1.401 & 1.359 & 0.975 \\
\hline HUKNAD & 1 & 1.283 & 1.45 & 1.407 & 1.355 & 0.965 \\
\hline IFENOX & 1 & 1.279 & 1.452 & 1.407 & 1.352 & 0.96 \\
\hline IGECOM & 1 & 1.279 & 1.448 & 1.401 & 1.346 & 0.87 \\
\hline IGERAO & 1 & 1.272 & 1.441 & 1.425 & 1.34 & 0.946 \\
\hline IGIKIT & 1 & 1.274 & 1.449 & 1.408 & 1.343 & 0.957 \\
\hline IHAREO & 1 & 1.299 & 1.419 & 1.414 & 1.316 & 0.942 \\
\hline IJOBEO & 1 & 1.269 & 1.448 & 1.392 & 1.355 & 1.152 \\
\hline IMOJAW & 1 & 1.279 & 1.446 & 1.398 & 1.362 & 0.82 \\
\hline INEXOP & 1 & 1.282 & 1.448 & 1.411 & 1.349 & 0.873 \\
\hline INEXUV & 1 & 1.29 & 1.453 & 1.404 & 1.352 & 0.88 \\
\hline INEYAC & 1 & 1.284 & 1.445 & 1.413 & 1.352 & 0.898 \\
\hline IROTOY & 1 & 1.275 & 1.443 & 1.39 & 1.354 & 0.821 \\
\hline ISEJIA & 1 & 1.285 & 1.455 & 1.398 & 1.354 & 0.918 \\
\hline ISEJIA & 2 & 1.289 & 1.451 & 1.401 & 1.358 & 0.964 \\
\hline ISENIE & 1 & 1.297 & 1.413 & 1.428 & 1.303 & 0.85 \\
\hline IVOHIL & 1 & 1.289 & 1.464 & 1.416 & 1.357 & 0.882 \\
\hline IVOHIL01 & 1 & 1.279 & 1.457 & 1.409 & 1.352 & 0.82 \\
\hline IZUYAE & 1 & 1.264 & 1.459 & 1.417 & 1.342 & 0.819 \\
\hline JOFFOA & 1 & 1.271 & 1.459 & 1.399 & 1.333 & 0.821 \\
\hline JOHKAU & 1 & 1.283 & 1.454 & 1.417 & 1.336 & 0.841 \\
\hline JUTKAK & 1 & 1.302 & 1.425 & 1.42 & 1.319 & 1.1 \\
\hline JUTKAK01 & 1 & 1.3 & 1.419 & 1.418 & 1.317 & 1.115 \\
\hline КАВМЕН & 1 & 1.277 & 1.457 & 1.397 & 1.343 & 0.82 \\
\hline KACPEL01 & 1 & 1.295 & 1.444 & 1.403 & 1.353 & 1.022 \\
\hline KACPEL01 & 2 & 1.295 & 1.444 & 1.408 & 1.342 & 1.016 \\
\hline KACPEL01 & 3 & 1.289 & 1.439 & 1.409 & 1.343 & 1.008 \\
\hline KAMKAL & 1 & 1.302 & 1.494 & 1.416 & 1.367 & 0.82 \\
\hline KASZAF & 1 & 1.283 & 1.441 & 1.418 & 1.355 & 1.03 \\
\hline KASZAF01 & 1 & 1.285 & 1.452 & 1.416 & 1.36 & 1.035 \\
\hline KAXPUV & 1 & 1.285 & 1.429 & 1.409 & 1.325 & 0.991 \\
\hline KAXPUV & 2 & 1.293 & 1.429 & 1.408 & 1.317 & 1.034 \\
\hline KEMROK & 1 & 1.161 & 1.383 & 1.416 & 1.217 & 0.82 \\
\hline KEZFUQ & 1 & 1.28 & 1.44 & 1.402 & 1.35 & 0.957 \\
\hline KIKNAU01 & 1 & 1.298 & 1.454 & 1.408 & 1.358 & 0.877 \\
\hline KIPMEB & 1 & 1.295 & 1.454 & 1.414 & 1.362 & 0.93 \\
\hline КIPMEB02 & 1 & 1.293 & 1.453 & 1.412 & 1.358 & 0.923 \\
\hline KIPTOU & 1 & 1.285 & 1.444 & 1.413 & 1.347 & 0.84 \\
\hline KOGVEH01 & 1 & 1.277 & 1.443 & 1.405 & 1.35 & 0.942 \\
\hline KULPOX & 1 & 1.274 & 1.453 & 1.39 & 1.366 & 0.82 \\
\hline KUPMIS & 1 & 1.276 & 1.456 & 1.406 & 1.338 & 0.82 \\
\hline
\end{tabular}




\begin{tabular}{|c|c|c|c|c|c|c|}
\hline KURDIL & 1 & 1.288 & 1.449 & 1.406 & 1.355 & 0.894 \\
\hline KURDIL & 2 & 1.286 & 1.453 & 1.408 & 1.346 & 0.924 \\
\hline KURWOK & 1 & 1.282 & 1.448 & 1.4 & 1.353 & 0.82 \\
\hline KUSJIS & 1 & 1.28 & 1.442 & 1.402 & 1.349 & 0.95 \\
\hline KUSJIS & 2 & 1.281 & 1.437 & 1.411 & 1.353 & 0.955 \\
\hline KUZWIM & 1 & 1.29 & 1.451 & 1.418 & 1.342 & 0.955 \\
\hline LANJAN & 1 & 1.263 & 1.449 & 1.39 & 1.343 & 0.815 \\
\hline LAPTIH & 1 & 1.273 & 1.46 & 1.406 & 1.34 & 0.82 \\
\hline LAQDEO & 1 & 1.278 & 1.459 & 1.395 & 1.325 & 0.84 \\
\hline LEFRIZ & 1 & 1.286 & 1.455 & 1.41 & 1.35 & 0.863 \\
\hline LEFRIZ & 2 & 1.284 & 1.453 & 1.406 & 1.353 & 0.879 \\
\hline LEFRIZ & 3 & 1.287 & 1.449 & 1.409 & 1.349 & 0.922 \\
\hline LEFRIZ & 4 & 1.287 & 1.453 & 1.407 & 1.35 & 0.905 \\
\hline LEFYOM & 1 & 1.277 & 1.443 & 1.39 & 1.343 & 0.85 \\
\hline LOFSOO & 1 & 1.29 & 1.438 & 1.402 & 1.336 & 1.17 \\
\hline LOJVUB01 & 1 & 1.28 & 1.434 & 1.411 & 1.349 & 0.959 \\
\hline LOMDUN & 1 & 1.284 & 1.451 & 1.408 & 1.354 & 0.973 \\
\hline LOMDUN & 2 & 1.285 & 1.449 & 1.405 & 1.355 & 0.976 \\
\hline LOMFAV & 1 & 1.287 & 1.445 & 1.412 & 1.355 & 0.907 \\
\hline LUJTOZ & 1 & 1.288 & 1.441 & 1.402 & 1.357 & 0.894 \\
\hline LUJTUF & 1 & 1.289 & 1.432 & 1.407 & 1.342 & 0.978 \\
\hline LUJVAN & 1 & 1.282 & 1.43 & 1.391 & 1.34 & 1.105 \\
\hline LUZKEW & 1 & 1.275 & 1.458 & 1.415 & 1.363 & 0.9 \\
\hline MALCUX & 1 & 1.271 & 1.449 & 1.381 & 1.363 & 0.82 \\
\hline MAPFOZ & 1 & 1.277 & 1.444 & 1.399 & 1.351 & 0.82 \\
\hline MAPFUF & 1 & 1.282 & 1.444 & 1.392 & 1.35 & 0.819 \\
\hline MAVNEE & 1 & 1.286 & 1.437 & 1.41 & 1.345 & 0.819 \\
\hline MAVNEE & 2 & 1.278 & 1.444 & 1.405 & 1.344 & 0.82 \\
\hline MAVNII & 1 & 1.302 & 1.443 & 1.455 & 1.372 & 0.82 \\
\hline MAVNII & 2 & 1.287 & 1.479 & 1.422 & 1.37 & 0.82 \\
\hline MAVNII & 3 & 1.306 & 1.433 & 1.455 & 1.369 & 0.819 \\
\hline MAVNII & 4 & 1.293 & 1.478 & 1.428 & 1.364 & 0.819 \\
\hline MEPWUA & 1 & 1.277 & 1.455 & 1.388 & 1.353 & 0.821 \\
\hline MEPWUA & 2 & 1.281 & 1.44 & 1.397 & 1.347 & 0.82 \\
\hline METJIF & 1 & 1.28 & 1.451 & 1.421 & 1.347 & 0.903 \\
\hline METJOL & 1 & 1.286 & 1.448 & 1.41 & 1.35 & 0.964 \\
\hline MEWFID & 1 & 1.317 & 1.391 & 1.437 & 1.279 & 0.853 \\
\hline MISWIV & 1 & 1.29 & 1.448 & 1.404 & 1.356 & 0.911 \\
\hline MISWIV & 2 & 1.293 & 1.45 & 1.412 & 1.351 & 0.909 \\
\hline MITNUZ & 1 & 1.284 & 1.45 & 1.412 & 1.353 & 0.805 \\
\hline MOPNAH & 1 & 1.276 & 1.456 & 1.403 & 1.359 & 0.929 \\
\hline MOPNAH01 & 1 & 1.281 & 1.452 & 1.405 & 1.353 & 0.982 \\
\hline MOPNAH03 & 1 & 1.285 & 1.457 & 1.408 & 1.359 & 0.882 \\
\hline MOPNAH05 & 1 & 1.285 & 1.456 & 1.408 & 1.359 & 0.871 \\
\hline MOSLUC & 1 & 1.284 & 1.442 & 1.421 & 1.319 & 1.246 \\
\hline MOSPOA & 1 & 1.279 & 1.444 & 1.409 & 1.341 & 0.821 \\
\hline
\end{tabular}




\begin{tabular}{|c|c|c|c|c|c|c|}
\hline MOSPOA & 2 & 1.275 & 1.454 & 1.399 & 1.354 & 0.82 \\
\hline MUCDIY & 1 & 1.272 & 1.442 & 1.401 & 1.345 & 0.984 \\
\hline MURQEW & 1 & 1.279 & 1.452 & 1.394 & 1.365 & 0.959 \\
\hline MURQEW & 2 & 1.29 & 1.455 & 1.389 & 1.34 & 0.821 \\
\hline NEBHEJ & 1 & 1.276 & 1.442 & 1.429 & 1.35 & 0.931 \\
\hline NEBHEJ & 2 & 1.295 & 1.419 & 1.395 & 1.365 & 0.931 \\
\hline NEQNON & 1 & 1.288 & 1.448 & 1.412 & 1.349 & 0.924 \\
\hline NEQYOZ & 1 & 1.284 & 1.453 & 1.411 & 1.347 & 0.841 \\
\hline NESTOV & 1 & 1.283 & 1.444 & 1.393 & 1.357 & 0.933 \\
\hline NESTOV & 2 & 1.292 & 1.434 & 1.374 & 1.364 & 0.942 \\
\hline NEZQAL & 1 & 1.282 & 1.442 & 1.414 & 1.342 & 1.008 \\
\hline NEZXIB & 1 & 1.276 & 1.446 & 1.391 & 1.345 & 0.821 \\
\hline NEZXUN & 1 & 1.279 & 1.462 & 1.402 & 1.356 & 0.82 \\
\hline NOCYOU & 1 & 1.308 & 1.42 & 1.428 & 1.315 & 0.95 \\
\hline NOGSOS & 1 & 1.282 & 1.471 & 1.411 & 1.301 & 0.929 \\
\hline NOGSUY & 1 & 1.28 & 1.461 & 1.426 & 1.281 & 0.931 \\
\hline NOQVIZ & 1 & 1.279 & 1.45 & 1.409 & 1.338 & 0.82 \\
\hline NOQYAU & 1 & 1.292 & 1.452 & 1.4 & 1.354 & 0.84 \\
\hline NORFUW & 1 & 1.286 & 1.452 & 1.407 & 1.358 & 0.96 \\
\hline NORKEL & 1 & 1.279 & 1.434 & 1.404 & 1.348 & 0.834 \\
\hline NORKEL & 2 & 1.277 & 1.445 & 1.397 & 1.347 & 0.798 \\
\hline NUGVOB & 1 & 1.276 & 1.458 & 1.41 & 1.339 & 1.026 \\
\hline NUNFOR & 1 & 1.29 & 1.42 & 1.406 & 1.324 & 0.828 \\
\hline NUPVIE & 1 & 1.312 & 1.425 & 1.431 & 1.307 & 0.819 \\
\hline NUTQUO & 1 & 1.292 & 1.436 & 1.399 & 1.321 & 0.965 \\
\hline OCECEG & 1 & 1.277 & 1.444 & 1.4 & 1.352 & 0.885 \\
\hline ОСЕКАК & 1 & 1.278 & 1.441 & 1.413 & 1.337 & 0.82 \\
\hline ODANOX & 1 & 1.272 & 1.45 & 1.401 & 1.347 & 0.819 \\
\hline ODANOX01 & 1 & 1.283 & 1.451 & 1.406 & 1.354 & 0.953 \\
\hline OFEKOA & 1 & 1.278 & 1.45 & 1.403 & 1.344 & 0.821 \\
\hline OGEFEM & 1 & 1.273 & 1.448 & 1.403 & 1.339 & 0.82 \\
\hline OGEFEM & 2 & 1.277 & 1.449 & 1.404 & 1.34 & 0.82 \\
\hline OMODEZ & 1 & 1.285 & 1.451 & 1.405 & 1.351 & 0.902 \\
\hline OPHSAL11 & 1 & 1.278 & 1.453 & 1.403 & 1.351 & 0.941 \\
\hline OPHSAL11 & 2 & 1.277 & 1.441 & 1.406 & 1.351 & 0.982 \\
\hline OVIJUZ & 1 & 1.28 & 1.448 & 1.397 & 1.349 & 0.773 \\
\hline PAJPEW & 1 & 1.276 & 1.445 & 1.402 & 1.356 & 0.985 \\
\hline PAJPEW & 2 & 1.279 & 1.445 & 1.403 & 1.346 & 0.955 \\
\hline PAQKEZ & 1 & 1.278 & 1.456 & 1.42 & 1.341 & 0.839 \\
\hline PAQKID & 1 & 1.282 & 1.453 & 1.417 & 1.344 & 0.96 \\
\hline PAQKOJ01 & 1 & 1.277 & 1.456 & 1.411 & 1.349 & 0.811 \\
\hline PAVFID & 1 & 1.283 & 1.452 & 1.406 & 1.353 & 0.931 \\
\hline PAVYOC & 1 & 1.267 & 1.454 & 1.404 & 1.345 & 0.82 \\
\hline PAVYOC & 2 & 1.273 & 1.457 & 1.399 & 1.347 & 0.82 \\
\hline PAXKOQ & 1 & 1.266 & 1.453 & 1.4 & 1.342 & 0.82 \\
\hline PAXKOQ & 2 & 1.259 & 1.462 & 1.393 & 1.347 & 0.82 \\
\hline
\end{tabular}




\begin{tabular}{|c|c|c|c|c|c|c|}
\hline PAXSIS & 1 & 1.291 & 1.447 & 1.408 & 1.35 & 0.82 \\
\hline PEYPIT & 1 & 1.286 & 1.442 & 1.396 & 1.358 & 0.802 \\
\hline PEYPOZ & 1 & 1.28 & 1.443 & 1.399 & 1.359 & 0.821 \\
\hline PIFKEV & 1 & 1.28 & 1.441 & 1.398 & 1.35 & 0.992 \\
\hline PIMRAG & 1 & 1.278 & 1.455 & 1.407 & 1.341 & 0.82 \\
\hline PIMREK & 1 & 1.276 & 1.453 & 1.409 & 1.338 & 0.82 \\
\hline PINWEQ & 1 & 1.281 & 1.445 & 1.407 & 1.35 & 0.82 \\
\hline PINWIU & 1 & 1.267 & 1.451 & 1.413 & 1.349 & 0.821 \\
\hline PINWIU & 2 & 1.274 & 1.457 & 1.409 & 1.349 & 0.82 \\
\hline PIZZIJ & 1 & 1.282 & 1.446 & 1.41 & 1.357 & 0.841 \\
\hline PONSUH & 1 & 1.285 & 1.449 & 1.4 & 1.35 & 0.82 \\
\hline POPJUA & 1 & 1.264 & 1.444 & 1.391 & 1.346 & 0.935 \\
\hline POPJUA & 2 & 1.274 & 1.454 & 1.4 & 1.347 & 0.987 \\
\hline POPJUA01 & 1 & 1.286 & 1.442 & 1.4 & 1.348 & 0.82 \\
\hline POSPET & 1 & 1.286 & 1.447 & 1.409 & 1.354 & 0.934 \\
\hline POVBUX & 1 & 1.263 & 1.424 & 1.436 & 1.332 & 0.842 \\
\hline PUDNOS & 1 & 1.288 & 1.449 & 1.414 & 1.35 & 0.84 \\
\hline PUGHUV & 1 & 1.285 & 1.439 & 1.396 & 1.347 & 0.82 \\
\hline PUGKAE & 1 & 1.278 & 1.453 & 1.4 & 1.352 & 0.929 \\
\hline PUJGIL & 1 & 1.271 & 1.453 & 1.396 & 1.351 & 0.958 \\
\hline PUJGIL & 2 & 1.281 & 1.432 & 1.404 & 1.342 & 0.965 \\
\hline PUQQEY & 1 & 1.272 & 1.446 & 1.395 & 1.349 & 0.819 \\
\hline QACTUL & 1 & 1.294 & 1.43 & 1.415 & 1.354 & 0.95 \\
\hline QADRUK & 1 & 1.288 & 1.437 & 1.386 & 1.359 & 0.96 \\
\hline QAKJET & 1 & 1.277 & 1.453 & 1.407 & 1.349 & 0.82 \\
\hline QECLOB & 1 & 1.275 & 1.455 & 1.402 & 1.346 & 0.858 \\
\hline QEDDEK & 1 & 1.277 & 1.452 & 1.405 & 1.347 & 0.82 \\
\hline QEDMOD & 1 & 1.291 & 1.426 & 1.41 & 1.324 & 0.821 \\
\hline QERMIJ & 1 & 1.297 & 1.445 & 1.399 & 1.348 & 1.128 \\
\hline QEVTER & 1 & 1.286 & 1.442 & 1.404 & 1.35 & 0.962 \\
\hline QEXLOV & 1 & 1.289 & 1.446 & 1.416 & 1.353 & 0.951 \\
\hline QEXLOV & 2 & 1.286 & 1.453 & 1.411 & 1.355 & 0.956 \\
\hline QIGPUR01 & 1 & 1.271 & 1.468 & 1.393 & 1.342 & 0.853 \\
\hline QIGPUR01 & 2 & 1.268 & 1.459 & 1.401 & 1.34 & 0.833 \\
\hline QIGQAY & 1 & 1.286 & 1.447 & 1.414 & 1.35 & 0.82 \\
\hline QIYTOJ & 1 & 1.274 & 1.45 & 1.427 & 1.328 & 0.788 \\
\hline QIYTOJ & 2 & 1.277 & 1.445 & 1.416 & 1.344 & 0.746 \\
\hline QOTRAT & 1 & 1.284 & 1.445 & 1.407 & 1.352 & 0.82 \\
\hline QOXTIH & 1 & 1.289 & 1.447 & 1.41 & 1.357 & 0.822 \\
\hline QOXTUT & 1 & 1.286 & 1.453 & 1.409 & 1.354 & 0.902 \\
\hline QOXVAB & 1 & 1.287 & 1.449 & 1.411 & 1.355 & 0.723 \\
\hline QOXVIJ & 1 & 1.291 & 1.449 & 1.408 & 1.357 & 0.962 \\
\hline QOXVIJ & 2 & 1.288 & 1.455 & 1.404 & 1.362 & 1.037 \\
\hline QOXVIJ01 & 1 & 1.282 & 1.456 & 1.393 & 1.362 & 0.973 \\
\hline QOXVIJ01 & 2 & 1.289 & 1.458 & 1.401 & 1.362 & 0.999 \\
\hline QOYGOB & 1 & 1.276 & 1.443 & 1.406 & 1.342 & 0.82 \\
\hline
\end{tabular}




\begin{tabular}{|c|c|c|c|c|c|c|}
\hline QOZZOV & 1 & 1.286 & 1.444 & 1.409 & 1.348 & 0.913 \\
\hline QUYMAZ & 1 & 1.269 & 1.444 & 1.423 & 1.334 & 0.821 \\
\hline RAJCEM & 1 & 1.284 & 1.451 & 1.406 & 1.351 & 0.838 \\
\hline RALFIV & 1 & 1.29 & 1.443 & 1.397 & 1.351 & 0.904 \\
\hline RAVTIR & 1 & 1.267 & 1.467 & 1.393 & 1.339 & 0.874 \\
\hline RAYZAU & 1 & 1.274 & 1.444 & 1.403 & 1.369 & 0.942 \\
\hline REGXEI & 1 & 1.291 & 1.44 & 1.392 & 1.343 & 0.87 \\
\hline REVBAV & 1 & 1.275 & 1.458 & 1.418 & 1.368 & 0.901 \\
\hline REZNEQ & 1 & 1.282 & 1.432 & 1.412 & 1.349 & 0.82 \\
\hline RIRYUN & 1 & 1.289 & 1.425 & 1.412 & 1.351 & 0.82 \\
\hline SABCAB & 1 & 1.277 & 1.449 & 1.405 & 1.332 & 0.82 \\
\hline SALAMP & 1 & 1.285 & 1.448 & 1.401 & 1.349 & 0.905 \\
\hline SALCAN01 & 1 & 1.28 & 1.455 & 1.419 & 1.352 & 1.02 \\
\hline SALCAN03 & 1 & 1.294 & 1.466 & 1.421 & 1.365 & 0.964 \\
\hline SANYIP01 & 1 & 1.297 & 1.455 & 1.406 & 1.351 & 0.871 \\
\hline SEJCIV & 1 & 1.288 & 1.449 & 1.416 & 1.357 & 0.82 \\
\hline SEJCIV & 2 & 1.279 & 1.45 & 1.417 & 1.357 & 0.821 \\
\hline SEJCOB & 1 & 1.275 & 1.447 & 1.405 & 1.346 & 0.819 \\
\hline SEJCOB & 2 & 1.279 & 1.444 & 1.405 & 1.352 & 0.821 \\
\hline SEVGUV & 1 & 1.285 & 1.439 & 1.409 & 1.351 & 0.966 \\
\hline SIDQEB & 1 & 1.311 & 1.41 & 1.429 & 1.297 & 1.089 \\
\hline SIMGOL & 1 & 1.274 & 1.453 & 1.403 & 1.338 & 0.821 \\
\hline SIPFEC & 1 & 1.291 & 1.434 & 1.413 & 1.32 & 1.192 \\
\hline SIPVAP & 1 & 1.274 & 1.445 & 1.397 & 1.356 & 0.82 \\
\hline SIPVAP & 2 & 1.285 & 1.443 & 1.415 & 1.34 & 0.82 \\
\hline SITNOZ & 1 & 1.279 & 1.434 & 1.4 & 1.343 & 0.82 \\
\hline SOGDUO & 1 & 1.279 & 1.452 & 1.425 & 1.349 & 0.825 \\
\hline SOHRIS & 1 & 1.285 & 1.452 & 1.405 & 1.346 & 0.851 \\
\hline SOLYEY & 1 & 1.281 & 1.442 & 1.402 & 1.35 & 0.819 \\
\hline SOTCIO & 1 & 1.277 & 1.46 & 1.407 & 1.344 & 0.881 \\
\hline SOXBEN & 1 & 1.285 & 1.448 & 1.41 & 1.359 & 0.988 \\
\hline SOXGAO & 1 & 1.28 & 1.451 & 1.392 & 1.356 & 0.82 \\
\hline TABMUF & 1 & 1.284 & 1.446 & 1.408 & 1.351 & 0.898 \\
\hline TABMUF & 2 & 1.288 & 1.445 & 1.406 & 1.356 & 0.961 \\
\hline TABMUF & 3 & 1.293 & 1.445 & 1.409 & 1.355 & 0.996 \\
\hline TABMUF & 4 & 1.285 & 1.447 & 1.41 & 1.351 & 0.913 \\
\hline TAHBOT & 1 & 1.297 & 1.434 & 1.413 & 1.332 & 1.18 \\
\hline TAHBUZ & 1 & 1.299 & 1.434 & 1.423 & 1.315 & 1.17 \\
\hline TAQCUK & 1 & 1.277 & 1.449 & 1.412 & 1.343 & 0.83 \\
\hline TEGGOD & 1 & 1.282 & 1.452 & 1.406 & 1.36 & 0.949 \\
\hline TEGGUJ & 1 & 1.286 & 1.454 & 1.407 & 1.351 & 0.87 \\
\hline TEJPAZ01 & 1 & 1.274 & 1.463 & 1.389 & 1.345 & 0.894 \\
\hline TEJPAZ01 & 2 & 1.275 & 1.452 & 1.391 & 1.353 & 1.039 \\
\hline TEJPAZ01 & 3 & 1.271 & 1.444 & 1.394 & 1.338 & 1.008 \\
\hline TEJPAZ01 & 4 & 1.275 & 1.441 & 1.408 & 1.352 & 1.294 \\
\hline TEJPAZ01 & 5 & 1.268 & 1.437 & 1.4 & 1.343 & 1.044 \\
\hline
\end{tabular}




\begin{tabular}{|c|c|c|c|c|c|c|}
\hline TEJPAZ01 & 6 & 1.277 & 1.461 & 1.384 & 1.36 & 0.885 \\
\hline TEJPAZ01 & 7 & 1.274 & 1.459 & 1.392 & 1.351 & 0.854 \\
\hline TEJPAZ01 & 8 & 1.271 & 1.439 & 1.386 & 1.348 & 1.176 \\
\hline TESQUF & 1 & 1.275 & 1.462 & 1.422 & 1.343 & 0.821 \\
\hline TIBNUO & 1 & 1.28 & 1.445 & 1.403 & 1.353 & 0.82 \\
\hline TIHBAO & 1 & 1.274 & 1.451 & 1.395 & 1.349 & 1.039 \\
\hline TIHBAO & 2 & 1.276 & 1.452 & 1.4 & 1.348 & 0.984 \\
\hline TIHFEW & 1 & 1.288 & 1.445 & 1.408 & 1.351 & 0.976 \\
\hline TIHFEW & 2 & 1.284 & 1.448 & 1.404 & 1.355 & 0.913 \\
\hline TOXFOB & 1 & 1.288 & 1.461 & 1.394 & 1.372 & 1.131 \\
\hline UDOJEC & 1 & 1.278 & 1.446 & 1.397 & 1.348 & 0.89 \\
\hline UFAMAQ & 1 & 1.279 & 1.442 & 1.407 & 1.357 & 0.82 \\
\hline ULELON & 1 & 1.274 & 1.434 & 1.419 & 1.341 & 0.82 \\
\hline ULELUT & 1 & 1.274 & 1.455 & 1.4 & 1.35 & 0.817 \\
\hline UNUWEG & 1 & 1.285 & 1.447 & 1.402 & 1.35 & 0.82 \\
\hline UQUBAK & 1 & 1.284 & 1.456 & 1.404 & 1.35 & 0.853 \\
\hline UWOVIM & 1 & 1.28 & 1.453 & 1.396 & 1.36 & 0.82 \\
\hline UWOVIM & 2 & 1.278 & 1.449 & 1.394 & 1.358 & 0.82 \\
\hline UZAYUQ & 1 & 1.286 & 1.449 & 1.413 & 1.349 & 0.829 \\
\hline UZAYUQ01 & 1 & 1.286 & 1.449 & 1.413 & 1.349 & 0.829 \\
\hline VACGAJ & 1 & 1.285 & 1.452 & 1.402 & 1.364 & 0.89 \\
\hline VAGXIL & 1 & 1.297 & 1.45 & 1.406 & 1.349 & 0.948 \\
\hline VARFAX & 1 & 1.283 & 1.437 & 1.398 & 1.355 & 0.819 \\
\hline VARFAX & 2 & 1.282 & 1.449 & 1.392 & 1.351 & 0.821 \\
\hline VARFAX01 & 1 & 1.278 & 1.445 & 1.408 & 1.35 & 0.82 \\
\hline VARFAX01 & 2 & 1.276 & 1.448 & 1.402 & 1.341 & 0.82 \\
\hline VATQUE & 1 & 1.278 & 1.447 & 1.414 & 1.345 & 0.924 \\
\hline VATRAL & 1 & 1.279 & 1.452 & 1.399 & 1.348 & 0.993 \\
\hline VATRAL & 2 & 1.268 & 1.452 & 1.4 & 1.351 & 1.027 \\
\hline VEFPED & 1 & 1.293 & 1.435 & 1.397 & 1.36 & 0.788 \\
\hline VEFPED & 2 & 1.284 & 1.43 & 1.412 & 1.352 & 0.836 \\
\hline VEFTEG & 1 & 1.292 & 1.452 & 1.408 & 1.365 & 0.97 \\
\hline VELSAH & 1 & 1.281 & 1.452 & 1.408 & 1.345 & 0.801 \\
\hline VENLIK & 1 & 1.284 & 1.448 & 1.403 & 1.359 & 0.899 \\
\hline VETJOU & 1 & 1.273 & 1.445 & 1.418 & 1.35 & 0.82 \\
\hline VIDDAO & 1 & 1.282 & 1.444 & 1.417 & 1.345 & 0.82 \\
\hline VIDVUA & 1 & 1.238 & 1.457 & 1.408 & 1.316 & 1.48 \\
\hline VIKQAJ & 1 & 1.288 & 1.456 & 1.42 & 1.342 & 0.84 \\
\hline VOBCEW & 1 & 1.283 & 1.447 & 1.399 & 1.355 & 0.819 \\
\hline VOBCEW & 2 & 1.279 & 1.45 & 1.402 & 1.342 & 0.821 \\
\hline VOBCIA & 1 & 1.256 & 1.45 & 1.385 & 1.336 & 0.819 \\
\hline VUXSUD & 1 & 1.296 & 1.433 & 1.411 & 1.352 & 0.825 \\
\hline WACNAR & 1 & 1.281 & 1.451 & 1.402 & 1.34 & 0.82 \\
\hline WEGVEK & 1 & 1.284 & 1.446 & 1.416 & 1.357 & 1.025 \\
\hline WIGGAW & 1 & 1.273 & 1.448 & 1.405 & 1.35 & 0.873 \\
\hline WIGGIE & 1 & 1.273 & 1.45 & 1.406 & 1.354 & 0.981 \\
\hline
\end{tabular}




\begin{tabular}{|c|c|c|c|c|c|c|}
\hline WIGXER & 1 & 1.284 & 1.437 & 1.397 & 1.336 & 0.853 \\
\hline WINPAL & 1 & 1.287 & 1.449 & 1.414 & 1.347 & 0.886 \\
\hline WINPAL & 2 & 1.281 & 1.455 & 1.413 & 1.346 & 1 \\
\hline WIZQIG & 1 & 1.277 & 1.45 & 1.396 & 1.349 & 0.82 \\
\hline WUFMEQ & 1 & 1.277 & 1.445 & 1.4 & 1.351 & 0.989 \\
\hline WUFMEQ & 2 & 1.277 & 1.449 & 1.398 & 1.349 & 1.009 \\
\hline WUKKUI & 1 & 1.289 & 1.448 & 1.397 & 1.343 & 0.917 \\
\hline WUPDER & 1 & 1.274 & 1.435 & 1.396 & 1.35 & 0.896 \\
\hline WUPDER & 2 & 1.284 & 1.448 & 1.399 & 1.334 & 0.878 \\
\hline WUPDER & 3 & 1.279 & 1.436 & 1.396 & 1.342 & 0.728 \\
\hline WUPDER & 4 & 1.282 & 1.439 & 1.391 & 1.361 & 0.854 \\
\hline WUPDER & 5 & 1.28 & 1.438 & 1.401 & 1.337 & 1.138 \\
\hline WUPDOB & 1 & 1.278 & 1.443 & 1.405 & 1.349 & 0.961 \\
\hline WUPDOB & 2 & 1.279 & 1.446 & 1.397 & 1.341 & 0.919 \\
\hline WUPDOB & 3 & 1.28 & 1.439 & 1.399 & 1.349 & 0.771 \\
\hline WUPDOB & 4 & 1.286 & 1.442 & 1.392 & 1.344 & 1.015 \\
\hline WUPDOB & 5 & 1.278 & 1.436 & 1.415 & 1.344 & 1.107 \\
\hline WUPFAP & 1 & 1.267 & 1.473 & 1.421 & 1.315 & 0.82 \\
\hline WUPFAP & 2 & 1.249 & 1.451 & 1.4 & 1.348 & 0.822 \\
\hline WUPFAP & 3 & 1.298 & 1.445 & 1.396 & 1.334 & 0.821 \\
\hline WUPFAP & 4 & 1.275 & 1.435 & 1.395 & 1.321 & 0.82 \\
\hline WUPFAP & 5 & 1.288 & 1.409 & 1.421 & 1.359 & 0.82 \\
\hline XAZQIZ & 1 & 1.287 & 1.451 & 1.405 & 1.352 & 0.794 \\
\hline XAZYAA & 1 & 1.283 & 1.439 & 1.4 & 1.354 & 0.988 \\
\hline XEBROM & 1 & 1.283 & 1.455 & 1.414 & 1.351 & 0.765 \\
\hline XEHLAY & 1 & 1.265 & 1.452 & 1.418 & 1.331 & 0.82 \\
\hline XERHIN & 1 & 1.283 & 1.455 & 1.412 & 1.357 & 1.004 \\
\hline XEYSOK & 1 & 1.293 & 1.427 & 1.405 & 1.324 & 0.82 \\
\hline XEZQEZ & 1 & 1.273 & 1.45 & 1.411 & 1.348 & 0.821 \\
\hline XIGNEI & 1 & 1.284 & 1.441 & 1.418 & 1.358 & 0.903 \\
\hline XOKKOY & 1 & 1.284 & 1.432 & 1.405 & 1.345 & 0.968 \\
\hline XOZJEC & 1 & 1.284 & 1.444 & 1.397 & 1.354 & 0.82 \\
\hline XUBNEO & 1 & 1.275 & 1.452 & 1.414 & 1.354 & 0.85 \\
\hline XUJVED & 1 & 1.28 & 1.436 & 1.412 & 1.376 & 0.911 \\
\hline YAGVAF & 1 & 1.292 & 1.449 & 1.411 & 1.352 & 0.86 \\
\hline YAGXUB & 1 & 1.276 & 1.448 & 1.399 & 1.35 & 0.82 \\
\hline YAWPIX & 1 & 1.284 & 1.454 & 1.416 & 1.349 & 0.9 \\
\hline YAWXUR & 1 & 1.286 & 1.45 & 1.407 & 1.353 & 0.84 \\
\hline YAXKUF & 1 & 1.28 & 1.452 & 1.407 & 1.355 & 0.887 \\
\hline YAXQUL & 1 & 1.277 & 1.461 & 1.41 & 1.353 & 0.84 \\
\hline YEHYAN & 1 & 1.282 & 1.437 & 1.422 & 1.328 & 0.841 \\
\hline YESLUE & 1 & 1.287 & 1.449 & 1.404 & 1.356 & 0.84 \\
\hline YEYQOJ & 1 & 1.302 & 1.438 & 1.427 & 1.356 & 0.964 \\
\hline YICHAT & 1 & 1.285 & 1.446 & 1.401 & 1.358 & 0.753 \\
\hline YICHAT & 2 & 1.283 & 1.452 & 1.397 & 1.358 & 0.685 \\
\hline YICPAB & 1 & 1.277 & 1.449 & 1.406 & 1.333 & 0.985 \\
\hline
\end{tabular}




\begin{tabular}{|c|c|c|c|c|c|c|}
\hline YIFLEF & 1 & 1.27 & 1.45 & 1.391 & 1.352 & 0.779 \\
\hline YIRJUF & 1 & 1.266 & 1.439 & 1.409 & 1.372 & 0.819 \\
\hline YIXLEX & 1 & 1.282 & 1.443 & 1.414 & 1.328 & 0.736 \\
\hline YOCRAK01 & 1 & 1.287 & 1.445 & 1.413 & 1.359 & 0.941 \\
\hline YODPEN & 1 & 1.276 & 1.448 & 1.397 & 1.355 & 0.883 \\
\hline YODXAS & 1 & 1.285 & 1.453 & 1.402 & 1.347 & 0.943 \\
\hline YODXAS01 & 1 & 1.281 & 1.449 & 1.39 & 1.355 & 0.995 \\
\hline YODXUM & 1 & 1.281 & 1.456 & 1.409 & 1.37 & 0.933 \\
\hline YODXUM01 & 1 & 1.275 & 1.452 & 1.407 & 1.368 & 0.949 \\
\hline YORFIU & 1 & 1.284 & 1.442 & 1.4 & 1.351 & 1.083 \\
\hline YOXGAT & 1 & 1.274 & 1.443 & 1.403 & 1.349 & 0.893 \\
\hline YUPYOY & 1 & 1.301 & 1.41 & 1.424 & 1.334 & 0.863 \\
\hline ZAMLUU & 1 & 1.303 & 1.435 & 1.397 & 1.344 & 1.25 \\
\hline ZAMMEF & 1 & 1.305 & 1.426 & 1.423 & 1.323 & 0.911 \\
\hline ZAQROY & 1 & 1.282 & 1.415 & 1.39 & 1.352 & 0.826 \\
\hline ZAQROY & 2 & 1.266 & 1.442 & 1.377 & 1.346 & 0.825 \\
\hline ZAQROY & 3 & 1.309 & 1.421 & 1.392 & 1.337 & 0.818 \\
\hline ZAQROY & 4 & 1.274 & 1.446 & 1.387 & 1.345 & 0.816 \\
\hline ZAWWAX & 1 & 1.283 & 1.453 & 1.413 & 1.346 & 0.82 \\
\hline ZAYNIW & 1 & 1.296 & 1.462 & 1.436 & 1.351 & 0.82 \\
\hline ZAYNIW & 2 & 1.295 & 1.429 & 1.391 & 1.373 & 0.82 \\
\hline ZEDDIV & 1 & 1.307 & 1.436 & 1.414 & 1.336 & 1.324 \\
\hline ZEDDIV01 & 1 & 1.316 & 1.419 & 1.422 & 1.319 & 1.181 \\
\hline ZEXPEX02 & 1 & 1.301 & 1.408 & 1.435 & 1.294 & 0.82 \\
\hline ZEXPEX02 & 2 & 1.307 & 1.405 & 1.428 & 1.298 & 0.819 \\
\hline ZIKNOW & 1 & 1.273 & 1.453 & 1.398 & 1.365 & 1.008 \\
\hline CHLSAN02 & 1 & 1.275 & 1.453 & 1.406 & 1.355 & 0.82 \\
\hline GOXLAI & 1 & 1.279 & 1.448 & 1.413 & 1.347 & 0.82 \\
\hline LOVDEH & 1 & 1.285 & 1.443 & 1.406 & 1.348 & 0.84 \\
\hline LOVDIL & 1 & 1.289 & 1.443 & 1.41 & 1.353 & 0.859 \\
\hline LOVDUX & 1 & 1.29 & 1.441 & 1.406 & 1.348 & 0.982 \\
\hline LUCMUT & 1 & 1.28 & 1.458 & 1.414 & 1.34 & 0.786 \\
\hline NOTYUS & 1 & 1.278 & 1.45 & 1.411 & 1.347 & 0.82 \\
\hline NOTYUS & 2 & 1.281 & 1.454 & 1.403 & 1.351 & 0.82 \\
\hline ROTHEP & 1 & 1.28 & 1.438 & 1.394 & 1.356 & 0.819 \\
\hline ROTHEP & 2 & 1.273 & 1.446 & 1.397 & 1.354 & 0.82 \\
\hline ROTREZ & 1 & 1.277 & 1.446 & 1.392 & 1.358 & 0.82 \\
\hline ROTREZ & 2 & 1.282 & 1.442 & 1.416 & 1.354 & 0.82 \\
\hline SOPFAG & 1 & 1.287 & 1.449 & 1.401 & 1.358 & 0.919 \\
\hline WOXYIT & 1 & 1.286 & 1.444 & 1.411 & 1.36 & 0.84 \\
\hline XOSNAW & 1 & 1.279 & 1.446 & 1.413 & 1.349 & 0.82 \\
\hline XOSNAW & 2 & 1.281 & 1.444 & 1.407 & 1.359 & 0.82 \\
\hline XOWCET & 1 & 1.286 & 1.445 & 1.403 & 1.353 & 0.834 \\
\hline average & & 1.282 & 1.446 & 1.406 & 1.347 & 0.892 \\
\hline
\end{tabular}


Table S6. Bond lengths of the six-membered pseudocycle in anils under the cis-keto form (31 retrieved structures; 38 total molecules). Chemical diagram for search is provided along with the table.

\begin{tabular}{|c|c|c|c|c|c|c|}
\hline & & & & $\begin{array}{l}R<5 \% \\
\text { No diso } \\
\text { structur }\end{array}$ & & \\
\hline NAME & $\begin{array}{c}\text { Number } \\
\text { of } \\
\text { molecules }\end{array}$ & $\begin{array}{c}\text { C7-N1 } \\
\text { (A) }\end{array}$ & $\begin{array}{c}\text { C7-C1 } \\
\text { (§) }\end{array}$ & $\begin{array}{c}\text { C1-C6 } \\
\text { (A) }\end{array}$ & $\begin{array}{c}\text { C6-01 } \\
(\AA)\end{array}$ & $\begin{array}{c}\text { N1-H1N } \\
(\AA)\end{array}$ \\
\hline BALGUR & 1 & 1.305 & 1.424 & 1.43 & 1.312 & 0.922 \\
\hline BALGUR01 & 1 & 1.301 & 1.423 & 1.43 & 1.312 & 0.952 \\
\hline CAQYIE & 1 & 1.303 & 1.407 & 1.412 & 1.298 & 1 \\
\hline CAQYIE & 2 & 1.296 & 1.399 & 1.419 & 1.302 & 0.997 \\
\hline CAQYIE01 & 1 & 1.304 & 1.403 & 1.425 & 1.298 & 1.022 \\
\hline СЕНМАF & 1 & 1.301 & 1.417 & 1.436 & 1.292 & 0.93 \\
\hline CIQCUB & 1 & 1.29 & 1.406 & 1.424 & 1.293 & 0.819 \\
\hline CIRTED & 1 & 1.317 & 1.388 & 1.435 & 1.306 & 0.877 \\
\hline CIRTED & 2 & 1.311 & 1.39 & 1.435 & 1.295 & 0.864 \\
\hline DUCWUU & 1 & 1.31 & 1.41 & 1.431 & 1.279 & 0.857 \\
\hline DUNXEQ & 1 & 1.311 & 1.413 & 1.443 & 1.271 & 0.856 \\
\hline DUSCEA & 1 & 1.309 & 1.409 & 1.456 & 1.258 & 0.88 \\
\hline DUSCEA & 2 & 1.308 & 1.41 & 1.456 & 1.265 & 0.88 \\
\hline EVOXIW01 & 1 & 1.299 & 1.428 & 1.422 & 1.322 & 1.154 \\
\hline EVOXIW01 & 2 & 1.308 & 1.417 & 1.429 & 1.3 & 0.998 \\
\hline FOCCOQ & 1 & 1.304 & 1.404 & 1.425 & 1.293 & 0.86 \\
\hline IFUMAZ & 1 & 1.311 & 1.416 & 1.435 & 1.298 & 1 \\
\hline KATKAS & 1 & 1.319 & 1.404 & 1.428 & 1.291 & 1.004 \\
\hline LIXRIT01 & 1 & 1.287 & 1.446 & 1.432 & 1.302 & 0.915 \\
\hline LIXRIT02 & 1 & 1.288 & 1.442 & 1.425 & 1.306 & 0.924 \\
\hline LIXRIT03 & 1 & 1.289 & 1.438 & 1.426 & 1.304 & 0.949 \\
\hline MOLQIO & 1 & 1.307 & 1.402 & 1.425 & 1.291 & 0.934 \\
\hline MOSTUK & 1 & 1.312 & 1.412 & 1.433 & 1.292 & 0.86 \\
\hline NEDMUF01 & 1 & 1.307 & 1.411 & 1.426 & 1.295 & 0.86 \\
\hline OLOHEC & 1 & 1.308 & 1.403 & 1.436 & 1.298 & 0.961 \\
\hline SAQTOT05 & 1 & 1.312 & 1.421 & 1.435 & 1.311 & 0.973 \\
\hline UQAVUE & 1 & 1.326 & 1.376 & 1.453 & 1.298 & 0.895 \\
\hline
\end{tabular}




\begin{tabular}{ccccccc}
\hline VAYJAG & 1 & 1.307 & 1.41 & 1.431 & 1.301 & 0.903 \\
VAYJAG & 2 & 1.302 & 1.413 & 1.433 & 1.299 & 0.928 \\
VAYJAG01 & 1 & 1.298 & 1.416 & 1.432 & 1.294 & 0.851 \\
VAYJAG01 & 2 & 1.299 & 1.41 & 1.438 & 1.297 & 0.868 \\
WOLYAX01 & 1 & 1.303 & 1.411 & 1.429 & 1.291 & 0.962 \\
XISJIT & 1 & 1.306 & 1.424 & 1.437 & 1.296 & 0.939 \\
YALTEK & 1 & 1.309 & 1.404 & 1.422 & 1.297 & 1.204 \\
YALTEK & 2 & 1.325 & 1.399 & 1.418 & 1.279 & 1.177 \\
YEFLOL & 1 & 1.297 & 1.412 & 1.438 & 1.279 & 1.02 \\
YIRQEW & 1 & 1.317 & 1.394 & 1.448 & 1.31 & 0.907 \\
YIRSIC & 1 & 1.298 & 1.41 & 1.443 & 1.276 & 0.86 \\
average & & $\mathbf{1 . 3 0 3}$ & $\mathbf{1 . 4 1 1}$ & $\mathbf{1 . 4 3 2}$ & $\mathbf{1 . 2 9 4}$ & $\mathbf{0 . 9 2 9}$
\end{tabular}

Table S7. Selected structures of SCC and MCC used for construction of model based on Hirshfeld surfaces C...C\% contacts calculation.

\begin{tabular}{|c|c|c|c|c|c|}
\hline $\begin{array}{c}\text { Structure } \\
\text { number }\end{array}$ & $\begin{array}{c}\text { Photo- }(\mathbf{P}) / \\
\text { Thermochromic (T) }\end{array}$ & $\mathrm{MCC} / \mathrm{SCC}$ & CSD refcode & C...C\% & T1 $\left(^{\circ}\right)$ \\
\hline 1 & $\mathrm{~T}$ & SCC & 1 , in this study & 2.7 & 32.2 \\
\hline 2 & $\mathrm{P}$ & MCC & 2 , in this study & 5.2 & 31.4 \\
\hline 3 & $\mathrm{P}$ & MCC & 3 , in this study & 2.3 & 40.6 \\
\hline 4 & $\mathrm{P}$ & MCC & 4, in this study & 5.2 & 31.6 \\
\hline 5 & $\mathrm{~T}$ & $\mathrm{SCC}$ & BADDAL12 1 & 6.1 & 5.9 \\
\hline 6 & $\mathrm{~T}$ & $\mathrm{SCC}$ & CAVQAR $^{1}$ & 5.5 & 6.06 \\
\hline 7 & $\mathrm{~T}$ & $\mathrm{SCC}$ & CAVQOF $^{2}$ & 6.3 & 7 \\
\hline 8 & $\mathrm{~T}$ & $\mathrm{SCC}$ & CULPOO $^{1}$ & 7.1 & 8.48 \\
\hline 9 & $\mathrm{P}$ & SCC & DAWFEN $^{3}$ & 2.2 & 41.58 \\
\hline 10 & $\mathrm{~T}$ & $\mathrm{SCC}$ & EKUGEW $^{4}$ & 6.8 & 13.54 \\
\hline 11 & $\mathrm{~T}$ & SCC & ETEYUX $^{5}$ & 7 & 9.92 \\
\hline 12 & $\mathrm{~T}$ & SCC & ETEYUX01 $^{6}$ & 5.4 & 13.46 \\
\hline 13 & $\mathrm{P}$ & SCC & FAXWIM $^{7}$ & 0.3 & 45.42 \\
\hline 14 & $\mathrm{P}$ & $\mathrm{SCC}$ & FAXWOS $^{7}$ & 1 & 46.1 \\
\hline 15 & $\mathrm{P}$ & SCC & FAXWUY $^{7}$ & 1.1 & 46.54 \\
\hline 16 & $\mathrm{~T}$ & $\mathrm{SCC}$ & GAWKEV $^{4}$ & 6.5 & 15 \\
\hline 17 & $\mathrm{~T}$ & SCC & GOFLAP $^{7}$ & 4.2 & 3.3 \\
\hline 18 & $\mathrm{~T}$ & SCC & GOKPAZ $^{1}$ & 8.5 & 3.2 \\
\hline 19 & $\mathrm{~T}$ & $\mathrm{SCC}$ & $\mathrm{HACZAN}^{1}$ & 7.1 & 9.82 \\
\hline 20 & $\mathrm{P}$ & $\mathrm{SCC}$ & IVOHIL $^{1}$ & 0.8 & 25.96 \\
\hline
\end{tabular}




\begin{tabular}{|c|c|c|c|c|c|}
\hline 21 & $\mathrm{~T}$ & $\mathrm{SCC}$ & KEZFUQ $^{1}$ & 7 & 4.67 \\
\hline 22 & $\mathrm{P}$ & $\mathrm{SCC}$ & KIPMEB $^{8}$ & 1.9 & 45.68 \\
\hline 23 & $\mathrm{P}$ & $\mathrm{MCC}$ & KIPTOU $^{9}$ & 0 & 17.81 \\
\hline 24 & $\mathrm{P}$ & $\mathrm{MCC}$ & KIPTOU $01^{10}$ & 0 & 21.5 \\
\hline 25 & $\mathrm{~T}$ & SCC & KOGVEH01 ${ }^{11}$ & 9.1 & 36.54 \\
\hline 26 & $\mathrm{~T}$ & SCC & KUFLUU $^{12}$ & 6.1 & 1.37 \\
\hline 27 & $\mathrm{~T}$ & $\mathrm{SCC}$ & KUFLUU01 ${ }^{12}$ & 4 & 26.94 \\
\hline 28 & $\mathrm{~T}$ & SCC & NADZUO $^{1}$ & 8.6 & 7.25 \\
\hline 29 & $\mathrm{~T}$ & MCC & $\mathrm{NEZXAT}^{13}$ & 7.9 & 11.08 \\
\hline 30 & $\mathrm{P}$ & $\mathrm{MCC}$ & $\mathrm{NEZXEX}^{13}$ & 1.2 & 41.58 \\
\hline 31 & $\mathrm{~T}$ & $\mathrm{SCC}$ & NEZXIB $^{13}$ & 7.4 & 6.68 \\
\hline 32 & $\mathrm{~T}$ & MCC & $\mathrm{NEZXOH}^{13}$ & 7.4 & 16.36 \\
\hline 33 & $\mathrm{P}$ & MCC & NEZXUN $^{13}$ & 1.8 & 45.01 \\
\hline 34 & $\mathrm{~T}$ & SCC & NEZYAU ${ }^{13}$ & 9.5 & 27.56 \\
\hline 35 & $\mathrm{~T}$ & $\mathrm{SCC}$ & NUQXAA $01^{14}$ & 4 & 2.48 \\
\hline 36 & $\mathrm{~T}$ & SCC & NUQXAA $02^{14}$ & 6.3 & 4.71 \\
\hline 37 & $\mathrm{~T}$ & SCC & NUQXAA03 ${ }^{14}$ & 7 & 2.12 \\
\hline 38 & $\mathrm{~T}$ & $\mathrm{SCC}$ & POFSEJ $^{1}$ & 8.1 & 3.93 \\
\hline 39 & $\mathrm{~T}$ & SCC & QOXVIJ01 ${ }^{15}$ & 3.2 & 39.82 \\
\hline 40 & $\mathrm{~T}$ & SCC & QOXVOP ${ }^{15}$ & 8 & 2.46 \\
\hline 41 & $\mathrm{P}$ & $\mathrm{MCC}$ & ROTHEP $^{5}$ & 0.7 & 3 \\
\hline 42 & $\mathrm{P}$ & $\mathrm{MCC}$ & ROTHIT $^{5}$ & 0.8 & 1.15 \\
\hline 43 & $\mathrm{P}$ & MCC & ROTREZ $^{5}$ & 0.7 & 0.67 \\
\hline 44 & $\mathrm{~T}$ & MCC & RUDLIN $^{10}$ & 4.2 & 10.68 \\
\hline 45 & $\mathrm{P}$ & MCC & RUDLOT $^{10}$ & 2.4 & 30.27 \\
\hline 46 & $\mathrm{P}$ & MCC & RUDMAG $^{10}$ & 0 & 23.74 \\
\hline 47 & $\mathrm{P}$ & MCC & RUDMEK $^{10}$ & 0 & 6.27 \\
\hline 48 & $\mathrm{P}$ & MCC & RUDMIO $^{10}$ & 0 & 14.73 \\
\hline 49 & $\mathrm{P}$ & SCC & SALCANO $^{1}$ & 1.4 & 47.72 \\
\hline 50 & $\mathrm{P}$ & SCC & SLCPYA $^{15}$ & 7.6 & 14.12 \\
\hline 51 & $\mathrm{P}$ & MCC & WIGFOJ $^{13}$ & 2.5 & 35.57 \\
\hline 52 & $\mathrm{P}$ & $\mathrm{MCC}$ & WIGFUP $^{13}$ & 5.2 & 35.12 \\
\hline 53 & $\mathrm{~T}$ & $\mathrm{MCC}$ & WIGGAW $^{13}$ & 3.5 & 9.09 \\
\hline 54 & $\mathrm{~T}$ & MCC & WIGGEA $^{13}$ & 4.2 & 15.09 \\
\hline 55 & $\mathrm{~T}$ & $\mathrm{MCC}$ & WIGGIE $^{13}$ & 4.2 & 8.53 \\
\hline 56 & $\mathrm{~T}$ & SCC & YOCRAK01 ${ }^{16}$ & 6.2 & 6.42 \\
\hline
\end{tabular}

1) Amimoto, K.; Kawato, T. Photochromism of organic compounds in the crystal state. J. Photochem. Photobiol. C. 2005, 6, 207-226. 
2) Burgess, J. O. H. N.; Fawcett, J.; Russell, D. R.; Gilani, S. R.; Palma, V. Four N-(2hydroxybenzylidene) aniline derivatives. Acta Crystallogr. Sect. C-Cryst. Struct. Commun. 1999, 55(10), 1707-1710.

3) Sliwa, M.; Létard, S.; Malfant, I.; Nierlich, M.; Lacroix, P. G.; Asahi, T.; Masuhara, H.; Yu. P.; Nakatani, K. Design, synthesis, structural and nonlinear optical properties of photochromic crystals: Toward reversible molecular switches. Chem. Mater. 2005, 17, 4727-4735.

4) Hadjoudis, E. Photochromic and thermochromic anils. Mol. Eng. 1995, 5, 301-337.

5) Hutchins, K. M.; Dutta, S.; Loren, B. P.; MacGillivray, L. R. Co-Crystals of a Salicylideneaniline: Photochromism Involving Planar Dihedral Angles. Chem. Mater. 2014, 26, 3042-3044.

6) Johmoto, K.; Ishida, T.; Sekine, A.; Uekusa, H.; Ohashi, Y. Relation between photochromic properties and molecular structures in salicylideneaniline crystals. Acta Crystallogr. Sect. B-Struct. Sci. 2012, 68, 297-304.

7) Sliwa, M.; Spangenberg, A.; Malfant, I.; Lacroix, P. G.; Métivier, R.; Pansu, R. B.; Nakatani, K. Structural, optical, and theoretical studies of a thermochromic organic crystal with reversibly variable second harmonic generation. Chem. Mater. 2008, 20(12), 4062-4068.

8) Harada, J.; Uekusa, H.; Ohashi, Y. X-ray analysis of structural changes in photochromic salicylideneaniline crystals. Solid-state reaction induced by two-photon excitation. J. Am. Chem. Soc. 1999. 121, 5809-5810.

9) Jacquemin, P. L.; Robeyns, K.; Devillers, M.; Garcia, Y. Reversible photochromism of an N-salicylidene aniline anion. Chem. Commun. 2014, 50, 649-651.

10) Jacquemin, P. L.; Robeyns, K.; Devillers, M.; Garcia, Y. Photochromism Emergence in N-Salicylidene p-Aminobenzenesulfonate Diallylammonium Salts. Chem. Eur. J. 2015, 21, 6832-6845.

11) Yüce, S.; Özek, A.; Albayrak, Ç.; Odabaşoğlu, M.; Büyükgüngör, O. A redetermination of 1-\{4-[(2-hydroxybenzylidene) amino] phenyl $\}$ ethanone. Acta Crystallogr. Sect. E.Struct Rep. Online. 2004, 60, o718-o719.

12) Carletta, A.; Dubois, J.; Tilborg, A.; Wouters, J. Solid-state investigation on a new dimorphic substituted N-salicylidene compound: insights into its thermochromic behaviour. CrystEngComm. 2015, 17, 3509-3518.

13) Johmoto, K.; Sekine, A.; Uekusa, H. Photochromism control of salicylideneaniline derivatives by acid-base co-crystallization. Cryst. Growth Des. 2012, 12, 4779-4786.

14)Zbačnik, M.; Nogalo, I.; Cinčić, D.; Kaitner, B. Polymorphism control in the mechanochemical and solution-based synthesis of a thermochromic Schiff base. CrystEngComm, 2015, 17, 7870-7877.

15) Robert, F.; Naik, A. D.; Tinant, B.; Robiette, R.; Garcia, Y. Insights into the Origin of Solid-State Photochromism and Thermochromism of N-Salicylideneanils: The Intriguing Case of Aminopyridines. Chem. Eur. J. 2009, 15, 4327-4342.

16) Avadanei, M.; Cozan, V.; Shova, S.; Paixão, J. A. Solid state photochromism and thermochromism of two related N-salicylidene anilines. Chem. Phys. 2014, 444, 43-51. 
